Published in final edited form as:

Nat Immunol. 2016 February ; 17(2): 169-178. doi:10.1038/ni.3318.

\title{
Group 3 innate lymphoid cells continuously require the transcription factor GATA3 after commitment
}

\author{
Chao Zhong ${ }^{1}$, Kairong Cui ${ }^{2}$, Christoph Wilhelm ${ }^{3,5}$, Gangqing $\mathrm{Hu}^{2}$, Kairui Mao ${ }^{4}$, Yasmine \\ Belkaid $^{3}$, Keji Zhao ${ }^{2}$, and Jinfang Zhu ${ }^{1, *}$ \\ ${ }^{1}$ Molecular and Cellular Immunoregulation Unit, Laboratory of Immunology, National Institute of \\ Allergy and Infectious Diseases, Bethesda, Maryland 20892 \\ ${ }^{2}$ Systems Biology Center, National Heart, Lung and Blood Institute, Bethesda, Maryland 20892 \\ ${ }^{3}$ Laboratory of Parasitic Diseases, National Institute of Allergy and Infectious Diseases, Bethesda, \\ Maryland 20892 \\ ${ }^{4}$ Laboratory of System Biology, National Institute of Allergy and Infectious Diseases, National \\ Institutes of Health, Bethesda, Maryland 20892 \\ ${ }^{5}$ Institute of Clinical Chemistry and Clinical Pharmacology, University Hospital Bonn, University of \\ Bonn, Bonn 53127, Germany
}

\section{Abstract}

The transcription factor GATA3 is indispensable for the development of all interleukin-7 receptor a (IL-7Ra)-expressing innate lymphoid cells (ILCs). However, the functional role of low GATA3 expression in committed ILC3s has not been identified. We report that GATA3 regulates homeostasis of ILC3s by controlling IL-7Ra expression. In addition, GATA3 is critical for the development of the NKp46 $6^{+}$ILC3 subset by regulating the balance between the transcription factors T-bet and ROR $\gamma$ t. Alhough GATA3 positively regulates NKp46 ${ }^{+}$ILC3 subset-specific genes, it negatively regulates CCR6 $6^{+}$ILC3 subset lymphoid tissue inducer (LTi)-specific genes in NKp $46^{+}$ILC3s. Furthermore, GATA3 is required for IL-22 production in both LTi and NKp46 ILC3s. Thus, despite its low expression, GATA3 is critical for the homeostasis, development and function of ILC3 subsets.

Users may view, print, copy, and download text and data-mine the content in such documents, for the purposes of academic research, subject always to the full Conditions of use:http://www.nature.com/authors/editorial_policies/license.html\#terms

*Correspondence: ; Email: jfzhu@ niaid.nih.gov, Phone: 301-402-6662, Fax: 301-480-7352

Accession numbers. RNA-Seq and ChIP-Seq data are available in the Gene Expression Omnibus (GEO) database (http:// www.ncbi.nlm.nih.gov/gds) under the accession number GSE71198.

AUTHOR CONTRIBUTIONS

C.Z. performed all the experiments; K.C. helped with constructing libraries for RNA-Seq and ChIP-Seq; C.W. helped with $C$. rodentium infection experiments; G.H. performed initial analyses of the RNA-Seq and ChIP-Seq data; K.M. performed imaging experiments; K.C., C.W., G.H., K.M., Y.B. and K.Z. offered suggestions to the project and edited the paper; C.Z. and J.Z. conceived the project, designed the experiments, analyzed the data and wrote the paper.

COMPETING FINANCIAL INTERESTS

The authors declare no competing financial interests. 
Interleukin-7 receptor a (IL-7Ra)-expressing innate lymphoid cells (ILCs) are regarded as the innate counterpart of the adaptive immune system's $C D 4^{+} T$ helper $\left(\mathrm{T}_{\mathrm{H}}\right)$ cells ${ }^{1}$. An especially strong parallel is the expression of master regulatory transcription factors and signature effector cytokines by distinct ILC and $\mathrm{T}_{\mathrm{H}}$ subsets. For example, type 2 ILCs (ILC2s) express the transcription factor GATA $3^{2},{ }^{3},{ }^{4}, 5$, which is the master regulator for type $2 \mathrm{~T}_{\mathrm{H}}\left(\mathrm{T}_{\mathrm{H}} 2\right)$ cells, and also secrete the prototypic $\mathrm{T}_{\mathrm{H}} 2$ cytokines IL-5 and IL-13 $6,{ }^{6}, 8$. Similarly, type 3 ILCs (ILC3s) express the transcription factor ROR $\gamma \mathrm{t}$ (encoded by the gene Rorc) and are capable of producing IL-17 and IL- $22^{9},{ }^{10},{ }^{11}$, as do $\mathrm{T}_{\mathrm{H}} 17$ cells. Finally, type 1 ILCs (ILC1s) ${ }^{12},{ }^{13}$ resemble T-bet-expressing $\mathrm{T}_{\mathrm{H}} 1$ cells whereas conventional natural killer (cNK) cells appear to be the innate counterpart of $\mathrm{CD}^{+} \mathrm{T}$ cells. Because of their capacity to produce similar cytokines, these functionally related ILCs and $\mathrm{T}_{\mathrm{H}}$ cells can participate in a specific type of immune response but at different stages ${ }^{12}, 14,15$; they also interact or crosstalk with each other through multiple mechanisms ${ }^{14},{ }^{16},{ }^{17}, 18,19$.

ILC3s consist of CCR6 $6^{+}$lymphoid tissue inducer (LTi) or LTi-like cells (we refer to these cells as CCR6 ${ }^{+}$ILC3s hereafter) and CCR6- ILC 3 lineages ${ }^{20}$. Some CCR6 ${ }^{-}$ILC3s express a natural cytotoxicity triggering receptor (NCR), NKp46 (encoded by the $N c r 1$ gene) in mouse ${ }^{9}$ and NKp44 (encoded by the NCR2 gene) in human ${ }^{21}$. CCR6 $6^{-}$ILC3s represent a separate ILC3 lineage that may eventually develop into $\mathrm{NCR}^{+} \mathrm{ILC}^{2} \mathrm{~s}^{20}$. Unlike LTi cells, CCR6 $^{-}$ILC3s are derived from PLZF ${ }^{+}$progenitors ${ }^{22}$ mainly after birth and they proliferate dramatically in number at $3 \sim 4$ weeks of age ${ }^{23}$. Although both $\mathrm{CCR}^{+}$and $\mathrm{NCR}^{+}$ILC3s are capable of producing IL-22 ${ }^{9},{ }^{11}$, a key cytokine that is essential for a protective immune response against intracellular bacteria such as Citrobacter rodentium, the unique function of $\mathrm{NCR}^{+}$ILC3s is still unclear.

In $\mathrm{T}$ cells, GATA3 not only regulates $\mathrm{T}_{\mathrm{H}} 2$ differentiation, but also directs $\mathrm{CD} 4^{+} \mathrm{T}$ cell development ${ }^{24}$. Similar to its function in $\mathrm{CD}^{+} \mathrm{T}$ cell development, GATA3 is indispensable for the development of all IL-7Ra-expressing $\mathrm{ILCs}^{3}$, including ILC3s ${ }^{25}$. GATA3 expression is high in ILC2s but it is also expressed at a low level in mature ILC1s and ILC3s. Although expressed at a low level, GATA3 is also required for maintaining ILC1 homeostasis ${ }^{12}$. However, it is unclear whether GATA3 has any function in mature ILC3s.

Here we show that deletion of Gata3 in already committed ILC3s by using Rorc-Cre blocks further development of CCR6 ${ }^{-} \mathrm{NCR}^{-}$ILC3s into $\mathrm{NCR}^{+}$ILC3s. Such arrest was partly due to up-regulation of ROR $\gamma$ t upon GATA3 removal. Furthermore, we found that GATA3 affected homeostasis and function of ILC3s by positively regulating the expression of IL-7Ra and IL-22, respectively. As a consequence, mice carrying a specific deletion of Gata3 in ILC3s succumbed to $C$. rodentium infection, which highlights the importance of maintained GATA3 expression in the ILC3 lineages.

\section{RESULTS}

\section{GATA3 affects ILC3 homeostasis via regulating IL-7Ra}

To study the function of GATA3 in already developed ILC3s, we crossed mice carrying floxed Gata3 alleles ${ }^{26}$ to the Rorc-Cre mice ${ }^{27}$ to generate conditional Gata3 deficient mice ( Gata $^{\mathrm{fl} / \mathrm{fl} 1}$ Rorc-Cre,referred to as Gata ${ }^{\mathrm{\Delta ILC} 3}$ hereafter) with GATA3 deficiency only in cells 
that had expressed and/or were expressing ROR $\gamma$ t, including ILC3s. Unlike Gata3 ${ }^{\text {fl/fl }}$ VavCre mice that do not have lymph node structure ${ }^{3}$, Gata $^{\mathrm{\Delta ILC} 3}$ mice developed lymph nodes suggesting that LTi cells were intact. Presumably because ROR $\gamma \mathrm{t}$ is also expressed during T cell development at the immature DP stage in the thymus ${ }^{27}$, and GATA3 is involved in CD4 ${ }^{+}$ $\mathrm{T}$ development ${ }^{24}$, we observed a modest reduction in $\mathrm{CD} 4^{+} \mathrm{T}$ cells (data not shown).

Since ROR $\gamma \mathrm{t}$ is not expressed in ILC progenitors such as common helper-like ILC progenitors (ChILPs) ${ }^{12}$ and PLZF $^{+}$common ILC progenitors ${ }^{22}$, Gata3 deletion in Gata ${ }^{\mathrm{ALC} 3}$ mice should not directly affect the development of ILC populations other than ILC3s. Indeed, GATA3 was abolished only in ILC3s but not in ILC2s (Supplementary Fig. 1a, b). ILC3s from the small intestine lamina propria (siLP) of Gata $^{\mathrm{AILC} 3}$ had a clear reduction of CD127 (IL-7Ra subunit) expression (Fig. 1a, b). CD127 expression was reduced in both $\mathrm{CCR}^{+}$and CCR6 ${ }^{-}$ILC3 subsets upon GATA3 deletion, but no substantial changes of CD90, Kit, Sca-1, CD25, and CD44 expression were noted (Supplementary Fig. 2a). One week after tamoxifen treatment of the $G a t a 3^{\mathrm{fl} / \mathrm{fl}} \mathrm{Cre}-\mathrm{ER}^{\mathrm{T} 2}$ mice, we also detected a similar reduction of CD127 expression in Gata3-deleted ILC3s (Fig. 1c) indicating that GATA3 is constantly reqired for regulating IL-7Ra expression in ILC3s. We then performed an RNA-Seq analysis comparing gene expression profiles between wild type and Gata3 ${ }^{\Delta I L C 3}$ ILC3s from siLP. II7r (encoding IL-7Ra) was among the list of genes whose expression was reduced in Gata3 ${ }^{\Delta \mathrm{ILC} 3}$ ILC3s compared to wild type ILC3s (Supplementary Fig. 2b).

IL-7 signaling is critical for the survival and proliferation of lymphocytes including ILCs. Indeed, the ratio of ILC3s to ILC2s was significantly reduced in Gata $3^{\mathrm{AILC} 3}$ mice compared to that in wild type mice (Fig. 1d). Furthermore, we found that the percentage as well as the absolute number of ILC3 cells was also reduced in Gata3 ${ }^{\mathrm{ILCC} 3}$ mice (Fig. 1e, f). Mixed bone marrow (BM) chimera experiments showed that alhthough B cells from either donor genotype of the chimeras populated normally in the irradiated $\mathrm{Rag}^{-/-} \mathrm{Il} \mathrm{rg}^{-/-}$recipients, Gata ${ }^{\mathrm{ALC} 3}$ ILC3s could not efficiently populate compared to wild type ILC3s (Supplementary Fig. 3a, b, c). These results indicate that the ILC3 cell number reduction in Gata3 deficient mice is a cell intrinsic effect.

We restored CD127 expression in Gata $3^{\mathrm{AILC} 3}$ ILC3 by crossing the Gata $3^{\mathrm{AILC} 3}$ mouse strain with a transgenic mouse line that carries an I17ra transgene driven by the human $C D 2$ promoter (Fig. 1g). In the mixed BM chimeras experiments, CD127 expression in ILC3s of different origin in the chimeric mice was identical to the CD127 expression in ILC3s found in their respective donors as expected (Fig. 1g, h). Transgenic II7ra efficiently restored CD127 in Gata3 ${ }^{\mathrm{ALC} 3}$ ILC3s, and rescued the ILC3 cell number to that observed in wild type chimeras (Fig. 1h, i). Therefore, GATA3 affects the homeostasis of ILC3s by regulating IL-7Ra expression.

We further performed anti-GATA3 chromatin immunoprecipitation (ChIP) followed by high throughput sequencing (ChIP-Seq) with ILC3s, ILC2s and $\mathrm{T}_{\mathrm{H}} 2$ cells. A common GATA3 binding peak located in the intron 2 of II7ra was identified in all three cell types (Fig. 1j). Three GATAA motifs were found within the GATA3 binding region. These results indicate that direct regulation of II7ra expression by GATA3 may be a shared mechanism for GATA3 mediated homeostatic regulation of both innate and adaptive lymphocytes. 


\section{GATA3 is indispensable for the development of NKp46+ ILC3s}

The expression of $N$ cr 1 was dramatically reduced in Gata $3^{\Delta I L C 3}$ ILC3s (Supplementary Fig. $2 b)$ suggesting that GATA 3 could play an important role during the development of $\mathrm{NKp} 46^{+}$ ILC3s. Indeed, although both CCR6 ${ }^{+}$and $\mathrm{CCR}^{-}$ILC3 lineages were present within the $\mathrm{ROR} \gamma \mathrm{t}^{+} \mathrm{ILC} 3 \mathrm{~s}$ in the Gata $^{\mathrm{AILC} 3}$ mice, the NKp46 ${ }^{+} \mathrm{ILC} 3$ population was drastically reduced (Fig. 2a).

We then further divided the CCR6- ILC3 lineage into three stages based on the expression of T-bet and NKp46: NKp46-T-bet ${ }^{-}$, NKp46-T-bet ${ }^{+}$and NKp46+T-bet ${ }^{+}$. Both the NKp46-T-

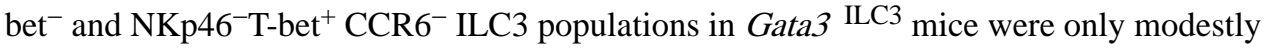
reduced in number similar to a reduction of CCR6 $6^{+}$ILC3s in these mice when compared to the respective populations in wild type mice (Fig. 2b), likely due to the decreased CD127. NKp $46^{+}$ILC3s were severely reduced in Gata $^{\mathrm{AILC} 3}$ mice (Fig. 2b, 2c). Furthermore, NKp46 expression was also reduced in the remaining Gata3-deficient NKp $46^{+}$ILC3s (Fig. 2d). ROR $\gamma$ t fate-mapping positive ILC1s (exILC3s) were also reduced in Gata3 ${ }^{\mathrm{AILC} 3}$ mice, while the ROR $\gamma$ t fate-mapping negative ILC1s were comparable between wild type and Gata $^{\mathrm{AILC} 3}$ mice (Supplementary Fig. 4a, b).

NKp $46^{+}$ILC3s are mainly enriched in the villi of the small intestine. Thus, we assessed the distribution of NKp46 $6^{+}$ILC3s by imaging the sections of the small intestines from wild type and Gata3 ${ }^{\mathrm{ILC} 3}$ mice. There was only a slight reduction of NKp46- ILC3s in the villi of the Gata $^{\mathrm{ALC} 3}$ mice compared to those in the wild type mice, whereas NKp $46^{+}$ILC3s were nearly absent in Gata3 ${ }^{\mathrm{ALC} 3}$ mice (Fig. 2e). NKp46 ${ }^{+} \mathrm{ILC} 3 \mathrm{~s}$ in tamoxifen-treated Gata ${ }^{\mathrm{fl} / \mathrm{fl}} \mathrm{Cre}-\mathrm{ER}^{\mathrm{T} 2}$ mice gradually decreased over time (Fig. 2f-h). Three months after tamoxifen treatment of the Gata ${ }^{\mathrm{fl} / \mathrm{fl}} \mathrm{Cre}-\mathrm{ER}^{\mathrm{T} 2}$ mice, NKp46 ${ }^{+}$ILC3s in these mice were dramatically reduced and similar to that observed in Gata $3^{\mathrm{AILC} 3}$ mice (Supplementary Fig. 5). Since ILC2s completely disappeared within 1 week of tamoxifen treatment whereas NKp $46^{+}$ILC3s were still present at a substantial number three weeks after Gata3 deletion, we conclude that GATA3 plays a minimal role in the maintenance of NKp46 $6^{+}$ILC3s and that the major function of GATA3 is to regulate the development of NKp46 ${ }^{+}$ILC3s. Restoration of CD127 in Gata3 ${ }^{\mathrm{ILC} 3}$ ILC3s by transgenic II7ra did not rescue the development of NKp46 ${ }^{+}$ILC3s (Fig. 2i) indicating that GATA3 determines the development of NKp46 ILC3s through a mechanism that is independent of CD127 regulation.

\section{Gata3 deficiency in ILC3s results in ROR $\gamma$ t upregulation}

The development of NKp46 ILC3s requires T-bet expression ${ }^{20},{ }^{28},{ }^{29}$. However, within the CCR6-NKp46- ILC3s, we did not detect notable changes in T-bet expression after Gata3 deletion at the population level (Fig. 3a). Unlike GATA3 binding to an intron of the Tbx21 gene (encoding T-bet) in ILC2 and $\mathrm{T}_{\mathrm{H}} 2$ cells, through which GATA3 could silence T-bet expression in these cells, GATA3 did not bind to the $T b \times 21$ locus in ILC3s, which may allow T-bet to be expressed in this lineage (Fig. 3b).

Consistent with increased Rorc mRNA after Gata3 deletion (Supplementary Fig. 2b), flow analysis also showed an increase in ROR $\gamma$ t protein expression in Gata3-deleted ILC3s (Fig. 3c, d). This was true in both CCR6 ${ }^{+}$and CCR6 ${ }^{-}$ILC3s (Supplementary Fig. 6a). Moreover, 
at the fetal stage, the Gata ${ }^{\mathrm{AILC} 3}$ LTi cells already had higher ROR $\gamma$ t than wild type LTi cells (Supplementary Fig. 6b). By using mixed BM chimeras, we confirmed that GATA3mediated suppression of ROR $\gamma \mathrm{t}$ expression was cell intrinsic (Supplementary Fig. 6c). Furthermore, we still detected an increased ROR $\gamma \mathrm{t}$ expression in ILC3s from the Gata ${ }^{\mathrm{IILC}}$ hCD2-II7ra mice, suggesting that GATA3 suppresses ROR $\gamma \mathrm{t}$ expression independently of CD127 regulation (Supplementary Fig. 6d). One week after tamoxifen treatment of the Gata $3^{\mathrm{fl} / \mathrm{fl}} \mathrm{Cre}-\mathrm{ER}^{\mathrm{T} 2}$ mice, ROR $\gamma \mathrm{t}$ expression was enhanced in ILC3s (Fig. $3 \mathrm{e})$, indicating that GATA3 constantly restrains ROR $\gamma$ t expression in mature ILC3s.

GATA3 directly binds to the Rorc gene in $\mathrm{T}_{\mathrm{H}} 2$ cells and regulatory $\mathrm{T}\left(\mathrm{T}_{\text {reg }}\right)$ cells ${ }^{30}$. Consistent with previous reports, GATA3 binding to the same site of the Rorc locus was found in ILC2s (Fig. 3f). A smaller but notable GATA3 binding peak at the same region was also detected in ILC3s, suggesting that GATA3 directly limits ROR $\gamma$ t expression in ILC3s.

\section{Interplay among ROR $\gamma \mathrm{t}$, GATA3 and T-bet at different stages}

To check whether a modest change in ROR $\gamma \mathrm{t}$ expression affects NKp46 ${ }^{+}$ILC3 development, we analyzed ROR $\gamma$ t heterozygous mice in which one allele of Rorc is replaced by $G f p$ $\left(\right.$ Rorc $\left.^{\mathrm{gfp} /+}\right)$. Although the Rorc ${ }^{\mathrm{gfp} /+}$ mice have been widely used as ROR $\gamma \mathrm{t}$ reporter mice and considered as "wild type" animals, compared to the real wild type littermates, these ROR $\gamma \mathrm{t}$ heterozygous mice had a dramatic increase of the NKp46 ${ }^{+}$ILC3s (Fig. 4a,b). Furthermore, Rorc ${ }^{\text {fpp/+ }}$ NKp46 ${ }^{+}$ILC3s also had higher NKp46 and T-bet expression compared to wild type NKp46 ${ }^{+}$ILC3s (Fig. 4c). These results indicate that a reduction in ROR $\gamma t$ expression by half promotes the development of $\mathrm{NKp} 46^{+}$ILC3s and T-bet expression in these cells.

To further understand the relationship between T-bet and ROR $\gamma \mathrm{t}$ in NKp46 ${ }^{+}$ILC3s, we generated a conditional ROR $\gamma$ t heterozygous mice by crossing $\operatorname{Rorc}{ }^{\mathrm{f} / /+}$ mice with $T b x 21$-Cre mice ( $\operatorname{Rorc}^{\mathrm{fl} /+} \mathrm{Tb} \times 21$-Cre). The proportion of NKp46 ${ }^{+}$ILC3s did not increase in these mice (Fig. 4d-e), unlike in Rorg ${ }^{\text {ffp } /+}$ mice, possibly due to a late deletion of the Rorc floxed allele by $T b \times 21$-Cre. Nevertheless, just as in Rorc ffp $/+^{\mathrm{NKp}} 46^{+}$ILC3s, a similar increase of NKp46 and T-bet expression was found in NKp46 ${ }^{+}$ILC3s from the Ror ${ }^{\mathrm{fl} /+}$ Tbx21-Cre mice compared to that from wild type littermates (Fig. 4f). These results suggest that the crossregulation and a dynamic balance between T-bet and ROR $\gamma \mathrm{t}$ may determine the fate of

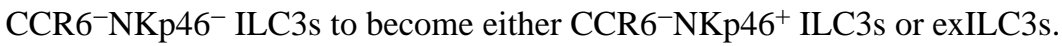

Taking advantage of a previously generated T-bet-ZsGreen reporter mouse ${ }^{31}$, we were able to analyze ROR $\gamma$ expression in CCR6 ${ }^{-} \mathrm{NKp}^{-} 6^{-} \mathrm{T}-$ bet-ZsG ${ }^{+}$cells in the presence or absence of T-bet. In the $T b \times 21^{-1-}$ T-bet-ZsGreen mice, although T-bet is not expressed due to $T b \times 21$ gene deletion, T-bet-ZsGreen expression in CCR6 $6^{-} \mathrm{NKp} 46^{-}$ILC3s was comparable between wild type and $T b \times 21$ deficient mice, indicating that at this early phase, T-bet was dispensable for its own expression (Fig. 4g, h). Importantly, ROR $\gamma \mathrm{t}$ in CCR6 ${ }^{-} \mathrm{NKp} 46^{-} \mathrm{T}-$ bet$\mathrm{ZsG}^{+}$were not increased in the absence of T-bet, suggesting that although T-bet suppresses ROR $\gamma$ t expression in NKp46 ${ }^{+}$ILC3s as reported earlier, it does not suppress ROR $\gamma \mathrm{t}$ expression in CCR6 $6^{-} \mathrm{NKp} 46^{-}$ILC3s (Fig. 4i). Since ROR $\gamma \mathrm{t}$ was increased in the Gata3 deficient cells, we conclude that ROR $\gamma \mathrm{t}$ is regulated by GATA3 but not T-bet at early developmental stages and that the interplay between GATA3 and ROR $\gamma$ t precedes the 
balance between T-bet and ROR $\gamma$ t, both of which are critical for the fate determination of the NKp46 ${ }^{+}$ILC3 lineage.

We corrected ROR $\gamma$ t expression in Gata3 ${ }^{\Delta \mathrm{ILC} 3}$ ILC3s by crossing these mice with Rorc ffp $/+^{\text {t }}$ heterozygous mice. ROR $\gamma \mathrm{t}$ expression in ILC3s from the resultant Gata $^{\mathrm{AILC} 3}$ Rorc $^{\mathrm{gfp} /+}$ mice was comparable to that in ILC3s from the wild type mice (Fig. 4j). More importantly, we observed a rescue of NKp46 $6^{+}$ILC3 development (Fig. 4k, 1). These results indicate that GATA3-mediated repression of ROR $\gamma \mathrm{t}$ in ILC3s at the early stages is critical for setting up an environment where the cross-regulation and balance between T-bet and ROR $\gamma \mathrm{t}$ can take place during the development of NKp46 $6^{+}$ILC3s.

\section{Lineage specific gene regulation by GATA3 in ILC3 subsets}

We further performed an RNA-Seq analysis of distinct ILC3 subsets. To obtain pure ILC3 subsets, we first generated an ROR $\gamma t$ reporter mouse strain carrying a transgenic bacterial artificial chromosome (BAC) containing the Rorc locus in which the DNA sequence of a fluorescent protein, E2-Crimson, was inserted at the start codon (ATG) of the ROR $\gamma \mathrm{t}$. In one of the transgenic lines, ROR $\gamma$ t-E2-Crimson-D9, E2-Crimson was highly expressed and faithfully reflected the expression of endogenous ROR $\gamma$ t (Fig. 5a). Unlike the Rorcffp/+ knock-in mice, our ROR $\gamma t$-E2-Crimson BAC transgenic mice had a normal distribution of ILC3 subsets (data not shown). The ROR $\gamma \mathrm{t}-\mathrm{E} 2-\mathrm{Crimson}$ reporter mice were then bred with the T-bet-ZsGreen reporter to generate a T-bet plus ROR $\gamma \mathrm{t}$ double reporter strain to facilitate sorting for the ILC3 subsets. Some of these double reporter mice were also bred onto the Gata $3^{\mathrm{ILC} 3}$ background. CCR6 $6^{+}$and NKp46 ${ }^{+}$ILC3 subsets defined by the expression of the reporters (ROR $\gamma \mathrm{t}$-E2-Crimson ${ }^{+} \mathrm{T}$-bet-ZsGreen ${ }^{-} \mathrm{CCR} 6^{+}$and ROR $\gamma \mathrm{t}$-E2-Crimson ${ }^{+} \mathrm{T}$-bet$\mathrm{ZsGreen}^{+} \mathrm{NKp} 46^{+}$for the CCR6 ${ }^{+}$and $\mathrm{NKp} 46^{+}$ILC3 populations, respectively, Fig. 5b) were then sorted from both wild type and Gata $3^{\mathrm{AICC} 3}$ mice and subjected to RNA-Seq analysis. Overall, GATA3 positively or negatively regulated (cutoff: $>2$ fold change of the genes with an RPKM value >5) hundreds of genes in either CCR6 $6^{+}$ILC3s (462 positively and 389 negatively) or NKp46 ${ }^{+}$ILC3s (841 positively and 751 negatively, Supplementary Table 1).

Using the same cutoff, we also identified hundreds of genes that were preferentially expressed by either CCR6 ${ }^{+}$ILC3s ( 420 genes) or NKp46 ${ }^{+}$ILC3s (377 genes). To further identify important genes that are most differentially expressed in $\mathrm{CCR}^{+}$and $\mathrm{NKp} 46^{+}$ ILC3s, we applied a more stringent cutoff: $>4$ fold change of the genes with an RPKM value $>10$, which resulted in $78 \mathrm{CCR}^{+}{ }^{+}$-ILC3-specific genes and $77 \mathrm{NKp} 46^{+}$-ILC3-specific genes (Supplementary Table 1 and Fig. 5c). CCR6 ${ }^{+}$-ILC3-specific genes included those encoding transcription factors such as Egr2, Foxs1, Id1 and Id3, and genes encoding cell surface molecules such as CXcr5, H2-Oa, Pdcd1, Ly6c1, Ly6c2, Nrp1, S1pr1 in addition to Ccr6. On the other hand, NKp46 ${ }^{+}$-ILC3-specific genes included those encoding transcription factors such as $H e y 1, I k z f 3, \operatorname{Irf} 7$ and $T b x 21$, and genes encoding cell surface molecules such as Ccr9, Cxcr3, Cxcr6, Fasl, Icos, II12rb1, Ltb4r1 in addition to Ncr1. CCR6 ${ }^{+}$ILC3s preferentially expressed $I I 17 f$, whereas NKp $46^{+}$ILC3s preferentially expressed $C s f 2$ and Areg, consistent with a previously reported finding ${ }^{32}$.

Among the 78 CCR6 $^{+}$-ILC3-specific genes and 77 NKp46+-ILC3-specific genes, 12 and 39 genes were positively regulated by GATA3 in these cells, respectively (Fig. 5d, e). Fifty out 
of the $78 \mathrm{CCR}^{+}$-ILC3-specific genes were negatively regulated by GATA3 in NKp46 ${ }^{+}$cells whereas 15 out of the $77 \mathrm{NKp} 46^{+}$-ILC3-specific genes were negatively regulated by GATA3 in $\mathrm{CCR}^{+}$cells (Fig. 5d, e). The fact that GATA3 promoted more than half of the NKp46+ILC3-specific genes, including II12rb1, Ltb4r1, Heyl, Ncrl, Ikzf3 and Icos, while inhibited more than half of the CCR6 ${ }^{+}$-ILC3-specific genes, including CXcr5, Id3, Egr2, Ly6c1 and Ly6c2, in NKp $46^{+}$ILC3s strongly supports the notion that GATA3 expression is particularly important for specifying the $\mathrm{NKp} 46^{+}$lineage fate.

\section{GATA3 regulates IL-22 production by ILC3s}

Among the 122 and 45 genes that were positively regulated by GATA3 ( $>4$ fold, RPKM>10) in CCR6 ${ }^{+}$and NKp46 ${ }^{+}$ILC3s, respectively, 9 genes, including Arg1 and II22, were induced by GATA3 in both cell types (Supplementary Table 1 and Fig. 6a). Furthermore, GATA3 positively regulated the expression of $A h r\left(>2\right.$ fold) in both CCR6 ${ }^{+}$ and NKp $46^{+}$ILC3s (Supplementary Table 1). Although IL-22 production by NKp $46^{+}$ILC3s has been correlated with the resistance of mice to Citrobacter rodentium infection, both $\mathrm{CCR}^{+}$and NKp46 ${ }^{+}$ILC3s are capable of producing IL-22. Indeed, our RNA-Seq results showed that both subsets expressed equivalent amounts of II22 mRNA, but II22 expression was dramatically reduced when GATA3 was absent in these cells (Fig. 6b). Our anti-GATA3 ChIP-Seq results showed that GATA3 bound to the promoter of the II22 gene only in ILC3s but not in ILC2s or $\mathrm{T}_{\mathrm{H}} 2$ cells (Fig. 6c). A GATA3-binding motif, AGATAA, was identified in the middle of the GATA3 binding region. Thus, $I 122$ is likely a direct target for GATA3 in both ILC3 subsets.

Compared to Gata $3^{\mathrm{fl} / \mathrm{fl}} \mathrm{ILC} 3 \mathrm{~s}$, Gata $^{\mathrm{AILC} 3} \mathrm{ILC} 3 \mathrm{~s}$ showed reduced IL-22 production by $\sim 50 \%$ in percentage (Fig. 6d). II7ra transgene restored the IL-7Ra expression in Gata $3^{\Delta I L C 3}$ ILC3s as shown above, but it did not correct the defect of such cells in producing IL-22 (Fig. $6 \mathrm{~d})$. In vitro stimulation by IL-23 induced IL-22 production in both wild type and Gata $3^{\mathrm{IILC} 3}$ ILC3s (Fig. 6d, 6e). However, IL-22 production was still substantially lower in Gata ${ }^{\mathrm{ALC} 3} \mathrm{ILC} 3 \mathrm{~s}$ than in wild type ILC3s.

To further address the importance of GATA 3 expression in ILC3s during immune responses, we used $C$. rodentium infection model. Since Gata $3^{\Delta \mathrm{ILC} 3}$ mice had somewhat altered T cell development as mentioned above, we bred these mice onto a Rag1 deficient background to

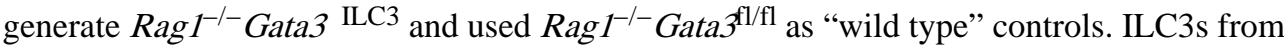
the Rag $^{-1-}$ Gata3 $^{\mathrm{AILC} 3}$ mice expressed less IL-7Ra but more ROR $\gamma$ t protein compared to ILC3s from Rag $1^{-/}$- Gata $3^{\mathrm{fl} / f 1}$ mice, as expected (Supplementary Fig. 7a). A blockage in NKp46 ${ }^{+}$ILC3 development was also observed in the Rag $^{-{ }^{-}-}$Gata $3^{\Delta I L C} 3$ mice (Supplementary Fig. 7b). Four days after infection, we detected a much lower frequency of ILC3s from Rag $^{-/-}$Gata ${ }^{\Delta \mathrm{ILC} 3}$ mice expressing IL-22 as compared to ILC3s from Rag $^{-1-}{ }^{-}$ata $3^{\mathrm{fl} / \mathrm{fl}}$ control mice (Fig. 6f). The total numbers of IL-22-producing ILC3s as well as the IL-22 MFI were significantly reduced in $\operatorname{Rag}^{-1-}$ Gata ${ }^{\mathrm{ALC} 3}$ mice compared to the control mice (Fig. 6g). We also detected reduced IL-22 but not IL-17a in the culture supernatant of $\mathrm{Rag}^{-1-}$ Gata3 $^{\mathrm{AILC} 3}$ colon lymphocytes (Fig. 6h). Consequently, Rag $1^{-1-}$ Gata3 $^{\Delta \mathrm{ILC} 3}$ mice experienced significant weigh loss starting from day 9 after infection (Fig. 6i). Although the vast majority of the $R a g 1^{-/}$Gata $3^{\mathrm{fl} / \mathrm{fl}}$ control mice survived 
infection at the end of the experiments, all the $\operatorname{Rag}^{-/-}$Gata $^{\mathrm{AILC} 3}$ mice died by 12 days after infection (Fig. 6j).

\section{DISCUSSION}

In this study, we have demonstrated that GATA3 is critical for the homeostasis of ILC3s, the development of NKp46 ${ }^{+}$ILC3s and IL-22 production by ILC3s.

IL-7Ra signaling is required for ILC development, survival and proliferation. IL-7Ra expression in ILC3s needs continuous expression of GATA3, and GATA3 binds to a region within intron 2 of the $I I 7 r$ gene. The binding pattern is very similar to that found in ILC3s, ILC2s and $\mathrm{T}_{\mathrm{H}} 2$ cells, although the expression of GATA3 is lower in ILC3s than in ILC2s and $\mathrm{T}_{\mathrm{H}} 2$ cells. This suggests that low GATA 3 expression may be necessary and sufficient for regulating IL-7Ra expression and that GATA3-mediated IL-7Ra expression may be a common mechanism through which GATA3 regulates the homeostasis of innate and adaptive lymphocytes.

Besides regulating ILC3 homeostasis, GATA3 is critical for the development of NKp46 ${ }^{+}$ ILC3s from CCR6 $6^{-} \mathrm{NKp} 46^{-}{ }^{-}$-bet $^{+}$ILC3s. Graded expression of T-bet is important for NKp $46^{+}$ILC3 lineage development and T-bet suppresses ROR $\gamma t$ expression in NKp46 ${ }^{+}$ ILC $3 \mathrm{~s}^{20}$. Here, we also report that ROR $\gamma \mathrm{t}$ suppresses T-bet expression in NKp46 $6^{+}$ILC3s. Therefore, the balance between T-bet and ROR $\gamma \mathrm{t}$ may determine NKp $46^{+}$ILC 3 fates. Although initial T-bet induction is not impaired in GATA3 deficient ILC3s, ROR $\gamma \mathrm{t}$ expression is enhanced in the absence of GATA3. On the other hand, T-bet deficiency abolishes the development of NKp $46^{+}$cells, ROR $\gamma t$ expression does not increase after T-bet deletion. These results indicate that there is a sequential involvement of GATA3 and T-bet in suppressing ROR $\gamma \mathrm{t}$ expression during the development of NKp46 ${ }^{+}$ILC3s.

ROR $\gamma$ t heterozygous mice have increased percentage of $\mathrm{NKp} 46^{+}$ILC3 compared to their wild type littermates. By correcting ROR $\gamma \mathrm{t}$ expression in Gata3 deficient ILC3s, the development of $\mathrm{NKp} 46^{+}$ILC3s was partially restored. These results suggest that GATA3 affects the balance between T-bet and ROR $\gamma$ t particularly at the early stage of NKp46 $6^{+}$ILC3 development and that sequential repression of ROR $\gamma$ t first by GATA3 and then by T-bet is necessary for the development and maturation of CCR6- ILC3s into NKp $46^{+}$cells. The delicate cross-regulation between T-bet, GATA3 and ROR $\gamma$ t at different stages offers a plausible explanation for why the development of NKp46 ${ }^{+}$ILC3s requires all three seemingly antagonistic transcription factors.

$\mathrm{CCR}^{+}{ }^{+} \mathrm{LTi}$ and NKp46 ${ }^{+}$ILC3s are two separate ILC3 populations and they develop from different progenitors ${ }^{22}$. Despite of their developmental and functional differences, only one study so far has assessed global gene expression in these cells ${ }^{32}$. Since ILC3s from the GFP knock-in ROR $\gamma$ t heterozygous reporter mice were used in the previous study, some findings may not truly reflect the gene expression in wild type ILC3s. Our results from using a double BAC T-bet plus ROR $\gamma \mathrm{t}$ reporter strain should better reflect the gene expression profiles in CCR6 $6^{+}\left(\mathrm{ROR} \gamma \mathrm{t}^{+} \mathrm{T}-\mathrm{bet}^{-}\right)$and $\mathrm{NKp}^{-} 6^{+}\left(\mathrm{ROR} \gamma \mathrm{t}^{+} \mathrm{T}-\mathrm{bet} \mathrm{t}^{+}\right)$ILC3 populations. Indeed, we not only observed a similar pattern of expression of most of the genes that were 
highlighted in the previous study, but also detected dozens of many lineage-specific genes that were not reported before. Thus, our datasets will be important for further understanding the biology as well as development of these two distinct ILC3 subsets.

Globally, GATA3 regulates hundreds of genes, either positively or negatively, in each ILC3 subset. Although more than half of the $\mathrm{NKp} 46^{+}$ILC 3 specific genes are positively regulated by GATA 3 in NKp $46^{+}$cells, the majority of the $\mathrm{CCR} 6^{+}$ILC 3 specific genes are negatively regulated by GATA3 in NKp $46^{+}$cells. This suggests that GATA3 may be an important switch in determining cell fate between $\mathrm{NKp}_{4} 6^{+}$and CCR6 $6^{+}$ILC3s, or possibly between $\mathrm{CCR}^{+}$and CCR6 ${ }^{-}$ILCs at earlier development stages. Interestingly, genes that are positively regulated by GATA 3 in one ILC 3 lineage could be negatively regulated by GATA3 in the other ILC3 lineage. All these results indicate that GATA3 regulates the expression of many lineage specific genes in these two ILC3 subsets in a cell-context dependent manner; GATA3 together with other lineage specific transcription factors, such as T-bet, Hey1, Ikzf3, Id3, Egr2 etc., many of which are newly identified in this study, form distinct transcriptional regulatory networks in defining ILC3 subsets.

Besides its effect on ILC3 homeostasis and NKp46 ${ }^{+}$ILC3 subset development, GATA3 also regulates their expression of the critical ILC3 effector cytokine IL-22. GATA3 directly binds to the promoter of the II22 gene only in ILC3s, but not in ILC2s or in $\mathrm{T}_{\mathrm{H}} 2$ cells. Besides directly regulating II22 transcription, GATA3 may also indirectly affect II22 expression by regulating the expression of $\mathrm{Ahr}$, a transcription factor critical for IL-22 production ${ }^{16,33}$. The relative contribution of these two mechanisms in IL-22 production requires further investigation. Consistent with all these phenotypical changes, mice with Gata3 deficiency in ILC3s are susceptible to $C$. rodentium infection.

In summary, although GATA3 is expressed at low levels in ILC3s, it plays a critical role in modulating the homeostasis, development and functions of the ILC3 subsets by directly regulating the expression of several important genes including II7r, Rorc and II22.

\section{METHODS}

Methods and any associated references are available in the online version of the paper.

\section{ONLINE METHODS}

\section{Mice}

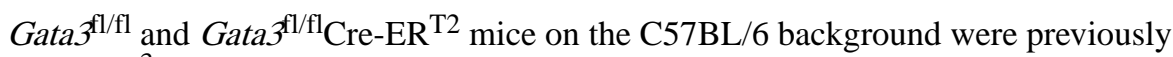
described ${ }^{3}$. To delete Gata3 in the Gata $3^{\mathrm{fl} / \mathrm{fl}} \mathrm{Cre}-\mathrm{ER}^{\mathrm{T} 2}$ mice, the mice were given intraperitoneal injection of $5 \mathrm{mg}$ tamoxifen in corn oil every other day for three times as previously described and analyzed on indicated days after the first injection. Gata $3^{\mathrm{fl} / \mathrm{fl}}$ mice were bred with the Rorc-Cre transgenic mice on the C57BL/6 background (a gift originally from Dr. Dan R. Littman of NYU, now also available at the Jackson Laboratory as Jax line 022791) to generate the Gata $3^{\Delta \mathrm{ILC} 3}$ mouse strain. Some Gata $3^{\mathrm{fl} / \mathrm{fl}}$ and $G a t a 3^{\Delta \mathrm{ILC} 3}$ mice were further bred with Tg- $h C D 2-I I 7 \mathrm{ra} \mathrm{mice}^{34}$ on the C57BL/6 background (kindly provided by Dr. Alfred Singer of NCI) or Rag $1^{-1-}$ mice (Taconic Line 146) to obtain Gata $3^{\text {fl/f1 }} h C D 2$ - 


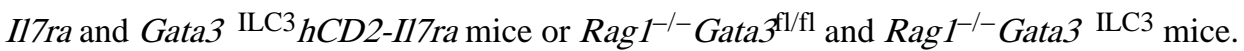
Some Rorc-Cre and Gata3 ${ }^{\mathrm{ILC} 3}$ mice were bred with the Rosa26 ${ }^{\text {tdTomato }}$ reporter mice (Jax line 007914), in which a loxP-STOP codon-loxP-tdTomato cassette was targeted to the ubiquitously expressed Rosa26 locus. In the resultant mice, tdTomato ${ }^{+}$cells represent the ROR $\gamma$ t-expressed and/or -expressing cells either in wild type (WT) and Gata ${ }^{\Delta \mathrm{ILC} 3}$ mice. Rorc-gfp KI mice (Jax line 007571), Rorc floxed mice (Jax line 008771) and Tbx21-Cre mice (a gift originally from Dr. Catherine Dulac of Harvard, now also available as Jax line 024507) were purchased from Jackson Laboratory. CD45.1 congenic mice (Taconic line 7) and $\mathrm{Rag}^{-/-} I 12 \mathrm{rg}^{-/-}$mice (Taconic line 111) were from the NIAID-Taconic repository. Tbet-ZsGreen reporter mice on wild type or $T b \times 21^{-1-}$ background were previously described $^{31}$. ROR $\gamma$ t reporter mice, ROR $\gamma$ t-E2-Crimson, were generated by bacterial artificial chromasome (BAC) transgenic strategy. Briefly, the coding region of a fluorescent protein E2-Crimson was amplified by PCR from vector plasmid pE2-Crimson-C1 (Clontech Laboratories, Inc.) and inserted at the ATG translational starting site of the coding sequence for ROR $\gamma \mathrm{t}$ in the BAC clone RP23-263I17 by recombineering technology. The primer sequences are: $5^{\prime}$ -

GCTGTCCTGGGCTACCCTACTGAGGAGGACAGGGAGCCAAGTTCTCAGTCATGGA TAGCACTGAGAACGTC-3' and 5'AGGACCCAGGCTCCCCCATGACCGGATGCCCCCATTCACTTACTTCTCATCAAACC ACAACTAGAATGCAGTG-3'.

The modified BAC clone was confirmed by sequencing and microinjected into fertilized C57BL/6 eggs to generate ROR $\gamma \mathrm{t}-\mathrm{E} 2-\mathrm{Crims}$ m mice. The founders of the transgenic mice were screened by Southern blot, PCR and FACS analysis for the intensity of E2-Crimson expression in peripheral blood lymphocytes. The offspring of one founder (D9) bearing a strong E2-Crimson fluorescent signal were selectively maintained. The ROR $\gamma$ t-E2-Crimson mice were genotyped by the following primers: E2-Crimson (F), 5'-

GATAGCACTGAGAACGTCATC-3' and E2-Crimson (R), 5'-

CCAGAGTCTTCTTCTGCATTA-3'. Some ROR $\gamma \mathrm{t}$-E2-Crimson mice were bred with Gata $^{31 / 1 / 1}$ T-bet-ZsGreen or Gata $3^{\Delta \mathrm{ILC} 3} \mathrm{~T}$-bet-ZsGreen mice to generate ROR $\gamma$ t and T-bet dual-reporter mice with or without Gata3 deletion in ILC3s. All mice were bred and/or maintained in the NIAID-specific pathogen-free animal facility. All experiments were done with mice of 6 to 16 weeks under protocols approved by the NIAID Animal Care and Use Committee.

\section{Citrobacter rodentium infection}

For C. rodentium (formerly Citrobacter freundii, biotype 4280) infections, strain DBS100 (kindly provided by Dr. David Artis, University of Pennsylvania, Philadelphia, Pennsylvania, USA) was prepared by selecting a single colony and cultured in LB broth for 8 hours. Mice were inoculated with approximately $1 \times 10^{10}$ colony forming units (CFU) in $200 \mu \mathrm{L}$ of PBS via oral gavage.

\section{Cell preparation and stimulation}

In the mixed bone marrow chimera experiments, total bone marrow cells were recovered from Taconic line 7, Gata $3^{\mathrm{fl} / \mathrm{fl}}$ and $G a t a 3^{\mathrm{ALC} 3}$, or other donors, and adoptively transferred 
into sublethally irradiated (450 Rad) $\mathrm{Rag}^{-/-} \mathrm{Il} 2 \mathrm{rg}^{-/-}$mice. For preparing and analyzing

ILCs, we used small intestine lamina propria (siLP) and colon lamina propria (cLP) with the following steps. Small intestine and colon were harvested from euthanized mice and the contents were emptied. Peyer's patches were removed from small intestine. Small intestine and colon were then opened longitudinally, cut into $1 \mathrm{~cm}$ pieces and shaken at $37{ }^{\circ} \mathrm{C}$ for 20 min in RPMI 1640 media containing 3\% FBS, 5 mM EDTA and 1 mM DTT followed by vortexed three times with RPMI 1640 media containing 2 mM EDTA to dissociate epithelial cells. The remained fragments were minced with scissor and digested at $37{ }^{\circ} \mathrm{C}$ for $30 \mathrm{~min}$ in RPMI 1640 media containing $0.1 \mathrm{mg} / \mathrm{ml}$ Liberase and $0.01 \%$ DNase I. The digested tissues were passed through a $40-\mu \mathrm{m}$ filter, centrifuged at $1600 \mathrm{rpm}$ for $6 \mathrm{~min}$ and resuspended in HBSS containing $3 \%$ FBS for further analysis.

For analysis of cytokine production by ILC3s, isolated siLP or cLP lymphocytes were resuspended by RPMI media containing $10 \%$ FBS and $2 \mathrm{ng} / \mathrm{ml}$ IL-7. In certain experiment as indicated, cells were also stimulated with $2 \mathrm{ng} / \mathrm{ml} \mathrm{IL}-23$. The cells were incubated at $37^{\circ} \mathrm{C}$ for 3 hours with the presence of $2 \mathrm{mM}$ monensin in the last 2 hours.

\section{Flow cytometry analysis}

Single-cell suspensions or stimulated cells were first incubated with anti-CD16/32 (clone 2.4G2) for 10 min to block Fc receptors. Cell surface molecules were then stained in HBSS with 3\% FBS followed by fixation. For intracellular staining of transcription factors, cells were fixed and permeabilized with Foxp3 staining buffer set purchased from eBioscience. For intracellular cytokine staining, cells were fixed in $1 \%$ formyl saline and permeabilized by $0.1 \%$ saponin buffer. Flow cytometry data were collected with LSR II (BD Biosciences) and the results were analyzed by FlowJo software (Tree Star).

Antibodies specific to mouse CD3 (2C11), CD5 (53-7.3), CD19 (eBio1D3), Gr-1 (RB68C5), CD45R (RA3-6B2), CD4 (RM4-5), NKp46 (29A1.4), Sca-1 (D7), CD25 (eBio3C7), CD45.1 (A20), CD45.2 (104), IL-22 (IL22JOP) were purchased from eBioscience; antibodies specific to mouse CD127 (A7R34), CCR6 (29-2L17), CD117 (2B8) were purchased from Biolegend; antibodies specific to mouse ROR $\gamma \mathrm{t}$ (Q31-378), GATA-3 (L50823), T-bet (O4-46) were purchased from BD Biosciences.

\section{Immunofluorescence staining}

Ileum part of small intestine was prepared as a "Swiss roll" and treated with the fixation and permeabilization solution (BD bioscience, 554722) for 12 hours followed by dehydrated in $30 \%$ sucrose prior to embedding in OCT freezing media (Sakura Finetek). $18 \mu \mathrm{m}$ sections were cut on a CM3050S cryostat (Leica) and adhered to Superfrost Plus slides (VWR). Frozen sections were permeabilized and blocked in PBS containing 0.1\% Triton X-100 (Sigma) and 10\% normal mouse serum (Jackson Immunoresearch) followed by staining in PBS containing $0.01 \%$ Triton X-100 and 5\% normal mouse serum. Mouse NKp46 was stained with polyclonal goat anti-mouse NKp46 serum (AF2225; R\&D Systems), followed by Alexa Fluor 555-conjugated donkey antibody to goat (anti-goat; A21432; Molecular Probes). ROR $\gamma$ t was stained with monoclonal anti-Human/Mouse ROR $\gamma$ t antibody (AFKJS-9; eBioscience), followed by Alexa Fluor 488-conjugated goat antibody to rat 
(anti-rat; A11006; Molecular Probes). Mouse epithelial cell adhesion molecule (EpCAM) and $\mathrm{CD} 3 \varepsilon$ was stained with rat anti-mouse EpCAM (G8.8; BD Pharmingen) and rat antimouse $\mathrm{CD} 3 \varepsilon$ (17A2; biolegend). After staining, slides are mounted with Fluormount $\mathrm{G}$ (Southern Biotech), and examined on a Leica TCS SP8 confocal microscope. Images were analyzed by Imaris software (Bitplane).

\section{RNA-Seq, ChIP-Seq and data analysis}

For total ILC3 RNA-Seq, live Lineage ${ }^{-} \mathrm{CD} 127^{+}$Thy $1{ }^{\text {high }}{ }^{-}$LRG-1 ${ }^{-}$cells were sorted from siLP of Gata $^{\text {fl/fl }}$ and Gata $3^{\Delta I L C 3}$ mice by FACSAria. For ILC3 subsets RNA-Seq, distinct ILC subsets were sorted from siLP of ROR $\gamma$ t-E2-Crimson plus T-bet-ZsGreen dual-reporter on the Gata $3^{\mathrm{fl} / \mathrm{fl}}$ or Gata3 ${ }^{\mathrm{\Delta ILC} 3}$ background. Total RNA was purified by QIAzol lysis reagent and miRNeasy Micro Kit (Qiagen). 10-20 ng of total RNA was amplified with the Ovation RNA-Seq System V2 (NuGEN). The double-stranded cDNA was then sonicated (30" on and 30 " off for 20 cycles) to an average size of 200 bp by Bioruptor Pico (Diagenode). Indexed sequencing libraries were then generated using $250 \mathrm{ng}$ sonicated cDNA with the KAPA LTP Library Preparation Kit (KAPABiosystems) according to the manufacture's protocol with minor modifications. Multiplex sequencing reads of $50 \mathrm{bp}$ were generated by the NHLBI DNA Sequencing and Computational Biology Core. Sequencing reads were mapped to the mm9 genome (UCSC Genome Browser). Gene expression levels in each sample were calculated by the RPKM (reads per kilobase of exon per million total reads) values. Differentially expressed genes were identified by edgeR with two cutoffs satisfying the following criteria: fold change $>4$, RPKM $>10$ in either sample or fold change $>2$, RPKM $>5$ in either sample.

For ChIP-Seq, live Lineage ${ }^{-} \mathrm{CD} 127^{+}$Thy $1{ }^{\text {high }}$ KLRG- $1^{-} \mathrm{NK} 1.1^{-}$ILC3s were sorted from siLP of $\mathrm{Rag} 1^{-1-}$ mice, live Lineage ${ }^{-} \mathrm{CD} 127^{+} \mathrm{Thy} 1^{+} \mathrm{KLRG}-1^{+}$ILC2s were sorted from mesenteric lymph nodes of IL-25-injected $R a g 1^{-1-}$ mice, and $\mathrm{T}_{\mathrm{H}} 2$ cells were prepared from three round differentiated naïve $\mathrm{CD} 4{ }^{+} \mathrm{T}$ cells as previous described ${ }^{31}$. Cells were crosslinked with $1 \%$ formaldehyde for $10 \mathrm{~min}$. Chromatin was prepared by sonication with Bioruptor Pico (30" on and 30" off for 9 cycles) and immunoprecipitated with anti-GATA3 (L50-823) and anti-mouse IgG-coated magnetic beads using iDeal ChIP-Seq Kit for transcription factors (Diagenode). ChIPed DNA was made into an indexed library and sequenced as described above for RNA-Seq.

\section{Statistics}

Samples were compared using Prism 6 software (GraphPad) by either a two-tailed unpaired Student's $t$-test or one-way or two-way ANOVA. A $P$ value of $<0.05$ was considered as significant.

\section{Supplementary Material}

Refer to Web version on PubMed Central for supplementary material. 


\section{ACKNOWLEDGMENTS}

We thank Drs. Avinash Bhandoola, Ronald Germain, John O'Shea, Pamela Schwartzberg and Alan Sher for their critical reading of our manuscript. We thank Dr. Liying Guo for her helpful suggestions and technical assistance in analyzing ILCs. We also thank Ms. Ke Weng for her assistance in cell sorting, Dr. Lionel Feigenbaum of NCI for his assistance in generating the ROR $\gamma \mathrm{t}$-E2-Crimson mice, and the NHLBI DNA Sequencing and Computational Biology Core for sequencing the RNA-Seq and ChIP-Seq libraries. The work was supported by the Intramural Research Program of NIH, NIAID and NHLBI.

\section{REFERENCES}

1. Spits H, Artis D, Colonna M, Diefenbach A, Di Santo JP, Eberl G, et al. Innate lymphoid cells--a proposal for uniform nomenclature. Nat Rev Immunol. 2013; 13(2):145-149. [PubMed: 23348417]

2. Hoyler T, Klose CS, Souabni A, Turqueti-Neves A, Pfeifer D, Rawlins EL, et al. The transcription factor GATA-3 controls cell fate and maintenance of type 2 innate lymphoid cells. Immunity. 2012; 37(4):634-648. [PubMed: 23063333]

3. Yagi R, Zhong C, Northrup DL, Yu F, Bouladoux N, Spencer S, et al. The transcription factor GATA3 is critical for the development of all IL-7Ralpha-expressing innate lymphoid cells. Immunity. 2014; 40(3):378-388. [PubMed: 24631153]

4. Klein Wolterink RG, Serafini N, van Nimwegen M, Vosshenrich CA, de Bruijn MJ, Fonseca Pereira D, et al. Essential, dose-dependent role for the transcription factor Gata3 in the development of IL-5+ and IL-13+ type 2 innate lymphoid cells. Proceedings of the National Academy of Sciences of the United States of America. 2013; 110(25):10240-10245. [PubMed: 23733962]

5. Mjosberg J, Bernink J, Golebski K, Karrich JJ, Peters CP, Blom B, et al. The transcription factor GATA3 is essential for the function of human type 2 innate lymphoid cells. Immunity. 2012; 37(4): 649-659. [PubMed: 23063330]

6. Moro K, Yamada T, Tanabe M, Takeuchi T, Ikawa T, Kawamoto H, et al. Innate production of T(H)2 cytokines by adipose tissue-associated c-Kit(+)Sca-1(+) lymphoid cells. Nature. 2010; 463(7280): 540-544. [PubMed: 20023630]

7. Neill DR, Wong SH, Bellosi A, Flynn RJ, Daly M, Langford TK, et al. Nuocytes represent a new innate effector leukocyte that mediates type-2 immunity. Nature. 2010; 464(7293):1367-1370. [PubMed: 20200518]

8. Price AE, Liang HE, Sullivan BM, Reinhardt RL, Eisley CJ, Erle DJ, et al. Systemically dispersed innate IL-13-expressing cells in type 2 immunity. Proceedings of the National Academy of Sciences of the United States of America. 2010; 107(25):11489-11494. [PubMed: 20534524]

9. Satoh-Takayama N, Vosshenrich CA, Lesjean-Pottier S, Sawa S, Lochner M, Rattis F, et al. Microbial flora drives interleukin 22 production in intestinal NKp46+ cells that provide innate mucosal immune defense. Immunity. 2008; 29(6):958-970. [PubMed: 19084435]

10. Buonocore S, Ahern PP, Uhlig HH, Ivanov II, Littman DR, Maloy KJ, et al. Innate lymphoid cells drive interleukin-23-dependent innate intestinal pathology. Nature. 2010; 464(7293):1371-1375. [PubMed: 20393462]

11. Sonnenberg GF, Monticelli LA, Elloso MM, Fouser LA, Artis D. CD4(+) lymphoid tissue-inducer cells promote innate immunity in the gut. Immunity. 2011; 34(1):122-134. [PubMed: 21194981]

12. Klose CS, Flach M, Mohle L, Rogell L, Hoyler T, Ebert K, et al. Differentiation of type 1 ILCs from a common progenitor to all helper-like innate lymphoid cell lineages. Cell. 2014; 157(2): 340-356. [PubMed: 24725403]

13. Fuchs A, Vermi W, Lee JS, Lonardi S, Gilfillan S, Newberry RD, et al. Intraepithelial type 1 innate lymphoid cells are a unique subset of IL-12- and IL-15-responsive IFN-gamma-producing cells. Immunity. 2013; 38(4):769-781. [PubMed: 23453631]

14. Halim TY, Steer CA, Matha L, Gold MJ, Martinez-Gonzalez I, McNagny KM, et al. Group 2 innate lymphoid cells are critical for the initiation of adaptive T helper 2 cell-mediated allergic lung inflammation. Immunity. 2014; 40(3):425-435. [PubMed: 24613091]

15. Basu R, O'Quinn DB, Silberger DJ, Schoeb TR, Fouser L, Ouyang W, et al. Th22 cells are an important source of IL-22 for host protection against enteropathogenic bacteria. Immunity. 2012; 37(6):1061-1075. [PubMed: 23200827] 
16. Qiu J, Guo X, Chen ZM, He L, Sonnenberg GF, Artis D, et al. Group 3 innate lymphoid cells inhibit T-cell-mediated intestinal inflammation through aryl hydrocarbon receptor signaling and regulation of microflora. Immunity. 2013; 39(2):386-399. [PubMed: 23954130]

17. Hepworth MR, Monticelli LA, Fung TC, Ziegler CG, Grunberg S, Sinha R, et al. Innate lymphoid cells regulate CD4+ T-cell responses to intestinal commensal bacteria. Nature. 2013; 498(7452): 113-117. [PubMed: 23698371]

18. Oliphant CJ, Hwang YY, Walker JA, Salimi M, Wong SH, Brewer JM, et al. MHCII-mediated dialog between group 2 innate lymphoid cells and CD4(+) T cells potentiates type 2 immunity and promotes parasitic helminth expulsion. Immunity. 2014; 41(2):283-295. [PubMed: 25088770]

19. Gasteiger G, Rudensky AY. Interactions between innate and adaptive lymphocytes. Nat Rev Immunol. 2014; 14(9):631-639. [PubMed: 25132095]

20. Klose CS, Kiss EA, Schwierzeck V, Ebert K, Hoyler T, d'Hargues Y, et al. A T-bet gradient controls the fate and function of CCR6-RORgammat+ innate lymphoid cells. Nature. 2013; 494(7436):261-265. [PubMed: 23334414]

21. Cella M, Fuchs A, Vermi W, Facchetti F, Otero K, Lennerz JK, et al. A human natural killer cell subset provides an innate source of IL-22 for mucosal immunity. Nature. 2009; 457(7230):722725. [PubMed: 18978771]

22. Constantinides MG, McDonald BD, Verhoef PA, Bendelac A. A committed precursor to innate lymphoid cells. Nature. 2014; 508(7496):397-401. [PubMed: 24509713]

23. Sawa S, Cherrier M, Lochner M, Satoh-Takayama N, Fehling HJ, Langa F, et al. Lineage relationship analysis of RORgammat+ innate lymphoid cells. Science. 2010; 330(6004):665-669. [PubMed: 20929731]

24. Wang L, Wildt KF, Zhu J, Zhang X, Feigenbaum L, Tessarollo L, et al. Distinct functions for the transcription factors GATA-3 and ThPOK during intrathymic differentiation of CD4(+) T cells. Nature immunology. 2008; 9(10):1122-1130. [PubMed: 18776904]

25. Serafini N, Klein Wolterink RG, Satoh-Takayama N, Xu W, Vosshenrich CA, Hendriks RW, et al. Gata3 drives development of RORgammat+ group 3 innate lymphoid cells. The Journal of experimental medicine. 2014; 211(2):199-208. [PubMed: 24419270]

26. Zhu J, Min B, Hu-Li J, Watson CJ, Grinberg A, Wang Q, et al. Conditional deletion of Gata3 shows its essential function in $\mathrm{T}(\mathrm{H}) 1-\mathrm{T}(\mathrm{H}) 2$ responses. Nature immunology. 2004; 5(11):11571165. [PubMed: 15475959]

27. Eberl G, Littman DR. Thymic origin of intestinal alphabeta T cells revealed by fate mapping of RORgammat+ cells. Science. 2004; 305(5681):248-251. [PubMed: 15247480]

28. Sciume G, Hirahara K, Takahashi H, Laurence A, Villarino AV, Singleton KL, et al. Distinct requirements for T-bet in gut innate lymphoid cells. The Journal of experimental medicine. 2012; 209(13):2331-2338. [PubMed: 23209316]

29. Rankin LC, Groom JR, Chopin M, Herold MJ, Walker JA, Mielke LA, et al. The transcription factor T-bet is essential for the development of NKp46+ innate lymphocytes via the Notch pathway. Nature immunology. 2013; 14(4):389-395. [PubMed: 23455676]

30. Wei G, Abraham BJ, Yagi R, Jothi R, Cui K, Sharma S, et al. Genome-wide analyses of transcription factor GATA3-mediated gene regulation in distinct T cell types. Immunity. 2011; 35(2):299-311. [PubMed: 21867929]

31. Zhu J, Jankovic D, Oler AJ, Wei G, Sharma S, Hu G, et al. The transcription factor T-bet is induced by multiple pathways and prevents an endogenous $\mathrm{Th} 2$ cell program during Th1 cell responses. Immunity. 2012; 37(4):660-673. [PubMed: 23041064]

32. Robinette ML, Fuchs A, Cortez VS, Lee JS, Wang Y, Durum SK, et al. Transcriptional programs define molecular characteristics of innate lymphoid cell classes and subsets. Nature immunology. 2015; 16(3):306-317. [PubMed: 25621825]

33. Qiu J, Heller JJ, Guo X, Chen ZM, Fish K, Fu YX, et al. The aryl hydrocarbon receptor regulates gut immunity through modulation of innate lymphoid cells. Immunity. 2012; 36(1):92-104. [PubMed: 22177117]

34. Yu Q, Erman B, Park JH, Feigenbaum L, Singer A. IL-7 receptor signals inhibit expression of transcription factors TCF-1, LEF-1, and RORgammat: impact on thymocyte development. The Journal of experimental medicine. 2004; 200(6):797-803. [PubMed: 15365098] 
a

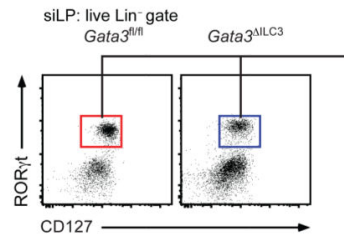

c

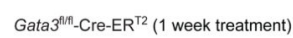

siLP: live Lin" gate

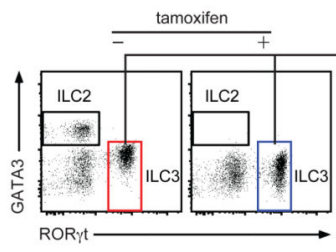

g

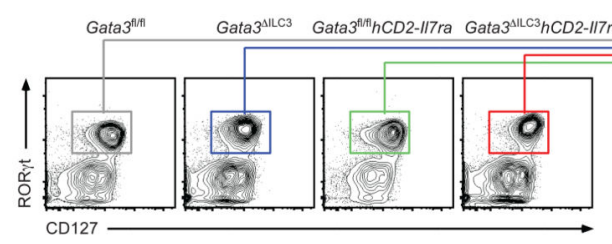

i

h

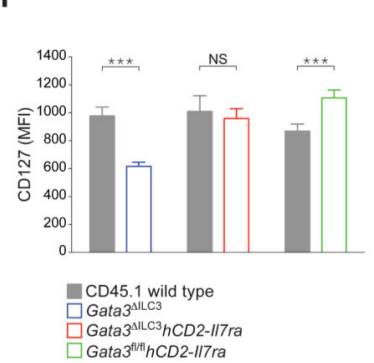

d

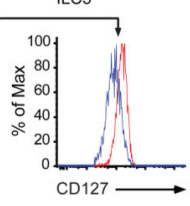

Chimerism

CD45.1: Wild type CD45.2: Gata ${ }^{\text {AlLC3 }}$
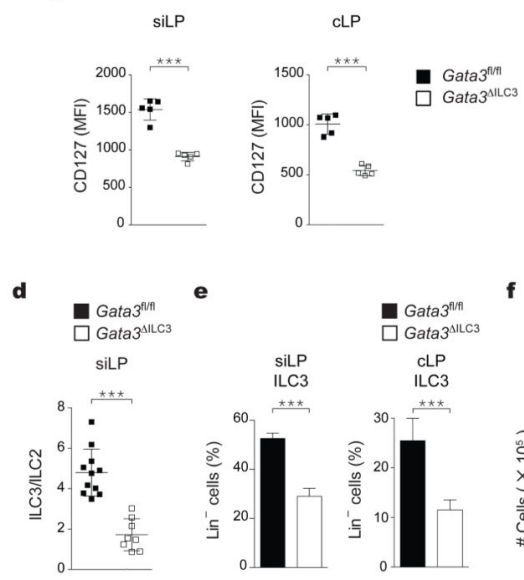

$\mathbf{f}$
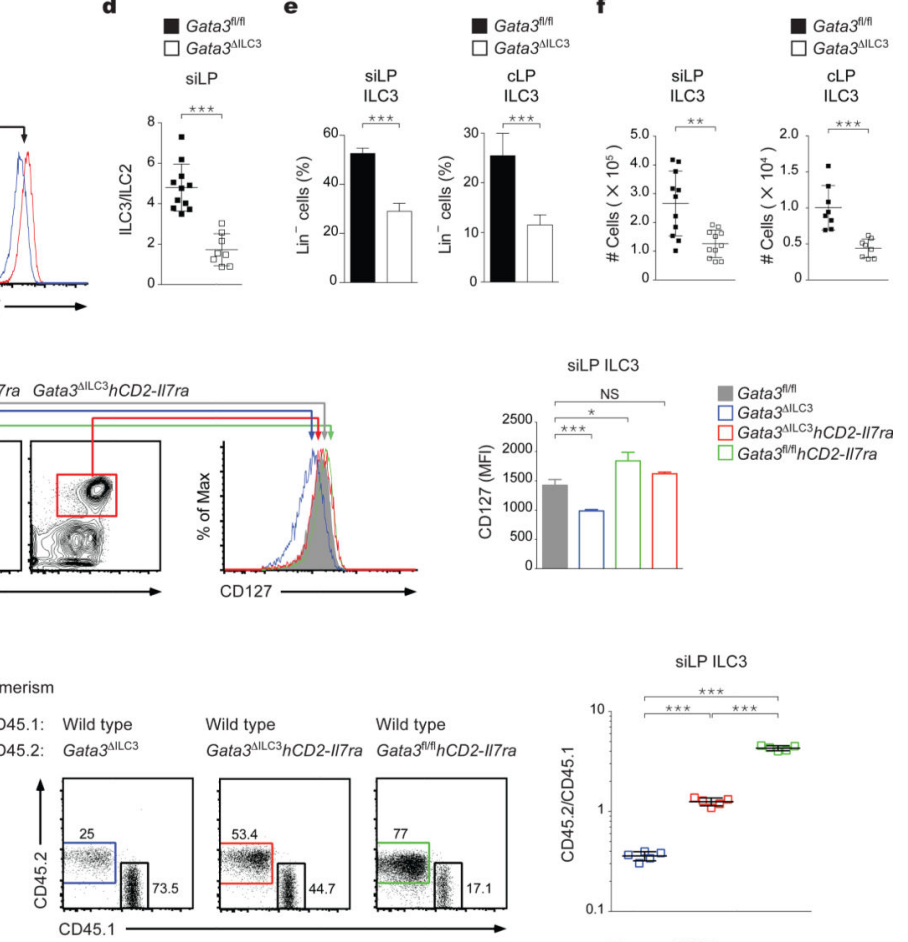

Wild type

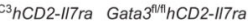

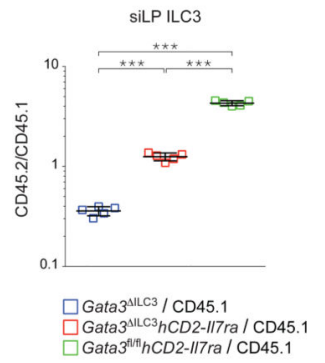

j

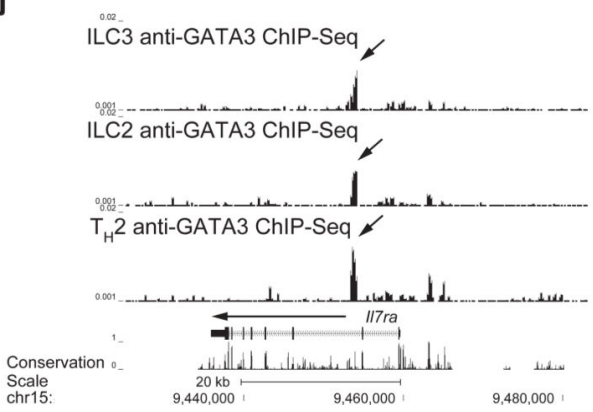

Figure 1.

GATA3 regulates IL-7Ra expression and ILC3 homeostasis. (a, b) Lymphocytes from small intestine lamina propria (siLP) of wild type (WT, Gata3 $\left.{ }^{\mathrm{fl} / \mathrm{fl}}\right)$ and Gata $^{\Delta \mathrm{ILC} 3}$ mice $(\mathrm{n}=5)$ were stained with anti-CD127 (IL-7Ra), lineage markers (CD3e, CD5, CD19, B220, and Gr-1) and ROR $\gamma$ t. CD127 expression on the ILC3s (live lineage ${ }^{-} \mathrm{CD} 127^{+} \mathrm{ROR} \gamma \mathrm{t}^{+}$) from siLP assessed by flow cytometry (a), and the mean fluorescent intensity (MFI) of CD127 expression by siLP or colon lamina propria (cLP) ILC3s (b). (c) Gata3 ${ }^{\mathrm{fl} / \mathrm{fl}} \mathrm{Cre}_{\mathrm{ER}} \mathrm{ER}^{\mathrm{T} 2}$ mice $(n=3)$ were treated with tamoxifen. One week later, lymphocytes from siLP were analyzed as 
in a. (d-f) Lymphocytes from siLP and cLP of the $\operatorname{Gata}^{\mathrm{fl} / \mathrm{fl}}(\mathrm{n}=11)$ and Gata $^{\Delta \mathrm{ILC} 3}$ mice $(\mathrm{n}=8)$ were stained as in $\mathbf{a}$. The ratio of ILC3s (live lineage ${ }^{-}$CD127 $\left.7^{+} \mathrm{ROR} \gamma \mathrm{t}^{+}\right)$to ILC2s (live lineage ${ }^{-} \mathrm{CD} 127^{+} \mathrm{GATA}^{+}$) was calculated (d). Percentages of ILC3s within the live lineage ${ }^{-}$ population (e), and total ILC3 cell numbers (f) in gut tissues are shown. (g) Lymphocytes from siLP of different mouse strains $(n=3)$ were analyzed as in $\mathbf{a}$. $(\mathbf{h}, \mathbf{i})$ Bone marrow (BM) cells from Gata3 ${ }^{\Delta \mathrm{ILC} 3}$, Gata $^{\mathrm{\Delta ILC} 3}$ hCD2-II7ra, or Gata3 ${ }^{\mathrm{fl} / \mathrm{fl}}$ hCD2-II7ra mice were cotransferred with congenic CD45.1 BM at 1:1 ratio into sublethally irradiated $\mathrm{Rag}^{-1-} \mathrm{II2 \textrm {rg } ^ { - 1 - }}$ recipients. CD127 level in ILC3s (h) and the composition of mixed ILC3s (i) in the siLP of chimeras ( $n=5)$ were analyzed 4-6 weeks later. (j) A UCSC (University of California, Santa Cruz) genome browser screen shot showing the normalized anti-GATA3 ChIP-Seq read density around $I I 7 r$ from different cells, with genomic region enriched with GATA3 binding highlighted by arrows. Each error bar represents s.d. of the mean. NS not significant, $* P<0.05, * * P<0.01, * * * P<0.001$ (two-tailed unpaired Student's $t$-test). Data are representative of at least three $(\mathbf{a}, \mathbf{b})$, two $(\mathbf{g}, \mathbf{h}, \mathbf{i})$ independent experiments and one experiment $(\mathbf{c}, \mathbf{j})$ or a combination of more than three experiments $(\mathbf{d}, \mathbf{e}, \mathbf{f})$. 


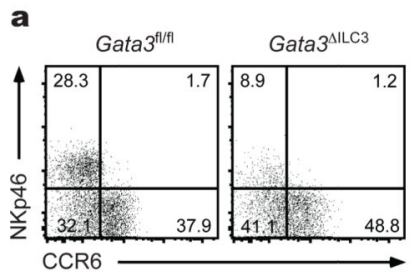

b

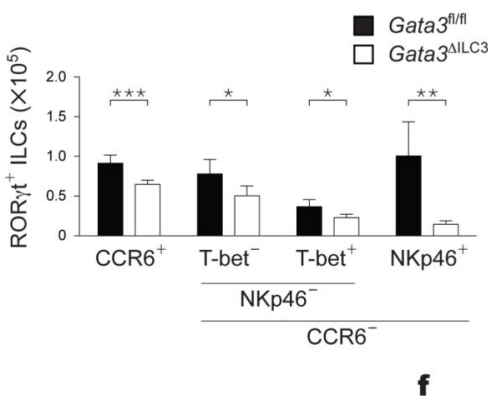

Intestine villi RORyt NKp46 CD3 EpCAM

Gata3 ${ }^{\text {flift }}$
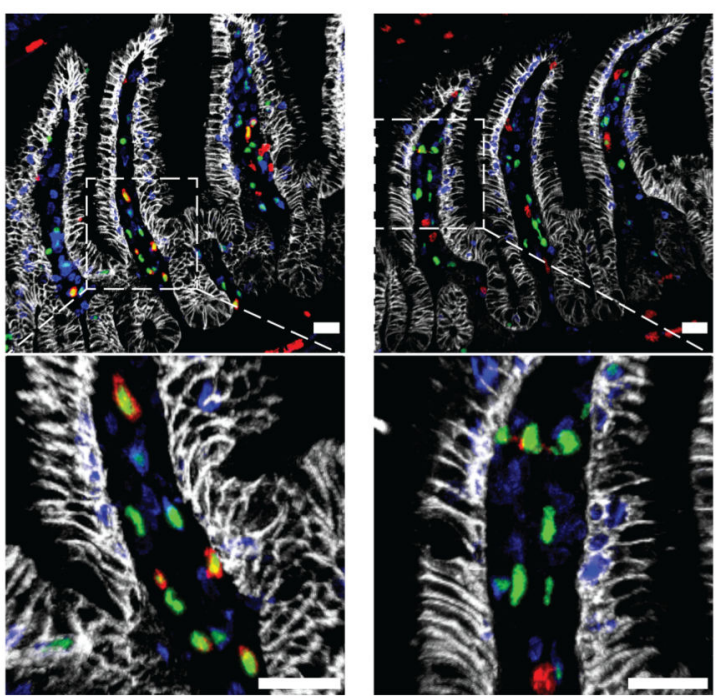

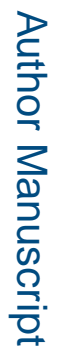

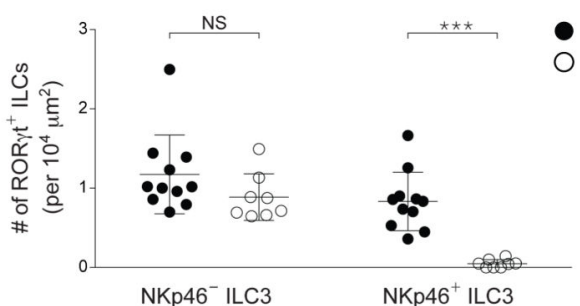

c

- ${\mathrm{NKp} 46^{+} \text {among CCR6 }}^{-}$ILC3 (\%)

Gata3 $^{\mathrm{fl/fl}} \quad \mathrm{Gata}^{\mathrm{ALLC3}}$
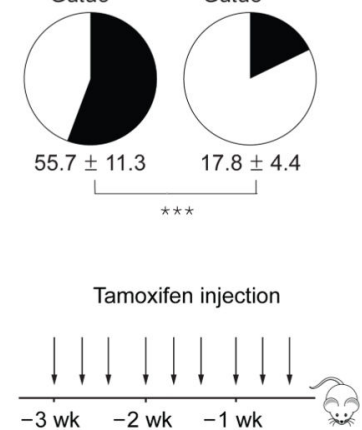

(Gata ${ }^{\mathrm{flff}} \mathrm{Cre}-\mathrm{ER}^{\mathrm{T}}{ }^{2}$ )

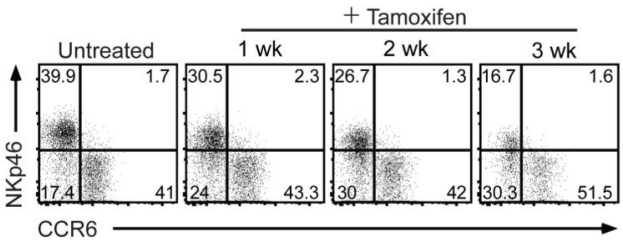

g

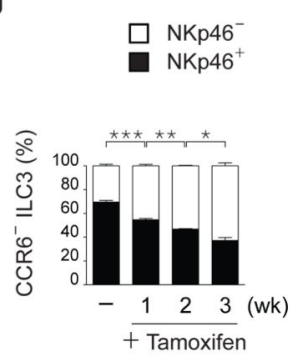

h

$\mathrm{CCR6}^{-} \mathrm{NKp} 46^{+}$

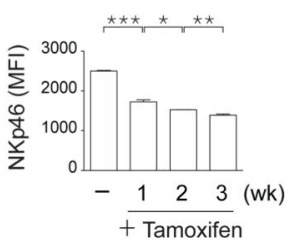

i

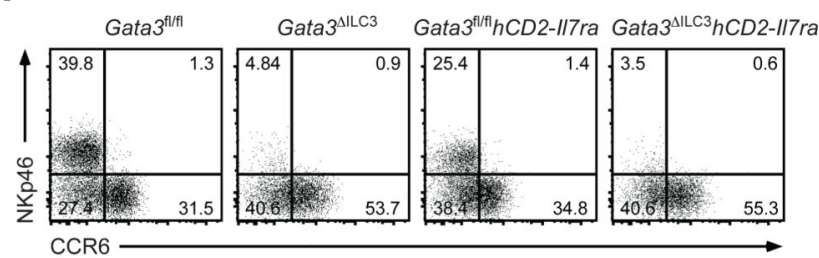

Figure 2.

GATA3 is indispensable for the development of NKp46 ${ }^{+}$ILC3s. (a-d) Lymphocytes from the siLP of the Gata $^{\mathrm{fl} / \mathrm{fl} 1}$ and $\operatorname{Gata}^{\mathrm{\Delta ILC} 3}$ mice (n>=3) were prepared and stained with live/ dead dye and antibodies to NKp46, CCR6, CD127, lineage markers, ROR $\gamma \mathrm{t}$ and T-bet. Different subsets of ILC3s (live lineage ${ }^{-} \mathrm{CD} 127^{+} \mathrm{ROR} \gamma \mathrm{t}^{+}$) were analyzed by flow cytometry (a). Absolute cell number of distinct ILC3 subsets in siLP (b). The percentage of NKp46 ILC3 within the CCR6- ILC3 lineage (c). The MFI of NKp46 expression within NKp46 ${ }^{+}$ ILC3s (d). (e) Distribution of NKp46 ${ }^{+}$ILC3s in small intestine villi was checked via imaging of the tissue sections after antibody staining. Frequency of appearance of indicated cell population within every $10^{4} \mu \mathrm{m}^{2}$ villi area in the section was counted and calculated. The white bars within the images reprent $25 \mu \mathrm{m}$ in length. (f-h) Gata $^{{ }^{\mathrm{f}} / \mathrm{fl} \mathrm{Cre}-\mathrm{ER}^{\mathrm{T} 2}}$ mice ( $n=3$ per group) were treated with tamoxifen every other day for three times to delete Gata3. 
3 weeks, 2 weeks, and 1 week after the first treatment, lymphocytes from siLP of these mice were analyzed as in $\mathbf{a}$. Untreated mice $(n=3)$ were used as control. Different subsets of ILC3s (live lineage ${ }^{-} \mathrm{CD} 127^{+} \mathrm{ROR} \gamma \mathrm{t}^{+}$) were analyzed by flow cytometry (f). Percentage of NKp46 ${ }^{+}$ILC3 within CCR6 ${ }^{-}$ILC3 lineage (g) and the MFI of NKp46 expression (h) at various time points after tamoxifen treatment were calculated. (i) Lymphocytes from siLP of indicated mice ( $\mathrm{n}=3$ per group) were stained as in a. Different subsets of ILC3s (live lineage ${ }^{-} \mathrm{CD} 127^{+} \mathrm{ROR} \gamma \mathrm{t}^{+}$) were analyzed by flow cytometry. Each error bar represents s.d. of the mean. NS not significant, $* P<0.05, * * P<0.01, * * * P<0.001$ (two-tailed unpaired Student's $t$-test). Data are representative of at least three $(\mathbf{a}, \mathbf{d})$, two $(\mathbf{e}, \mathbf{i})$ independent experiments and one experiment $(\mathbf{f}, \mathbf{g}, \mathbf{h})$ or a combination of at least three experiments $(\mathbf{b}$, c). 

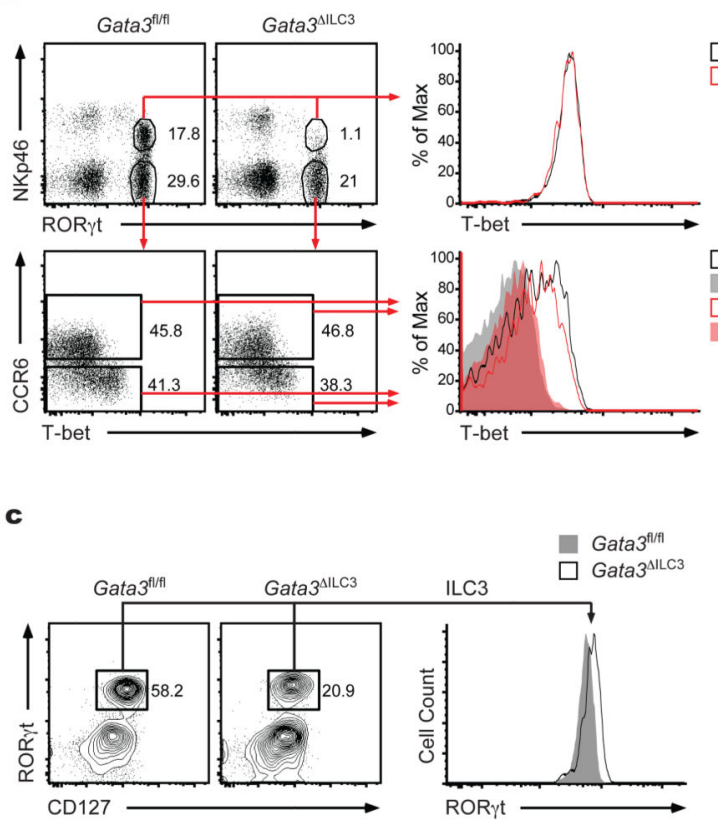

e $\quad\left(\right.$ Gata $\left.^{\mathrm{flff}} \mathrm{Cre}-\mathrm{ER}^{\mathrm{T}}\right)$

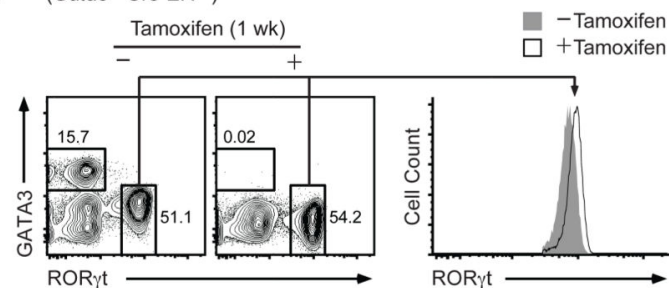

b

ILC3 anti-GATA3 ChIP-Seq

$\square$ Gata $^{\mathrm{fl/fl}} \mathrm{NKp} 46^{+}$ILC3

$\square$ Gata3 ${ }^{\Lambda L C C 3} \mathrm{NKp}^{\mathrm{N}} 6^{+}$ILC3

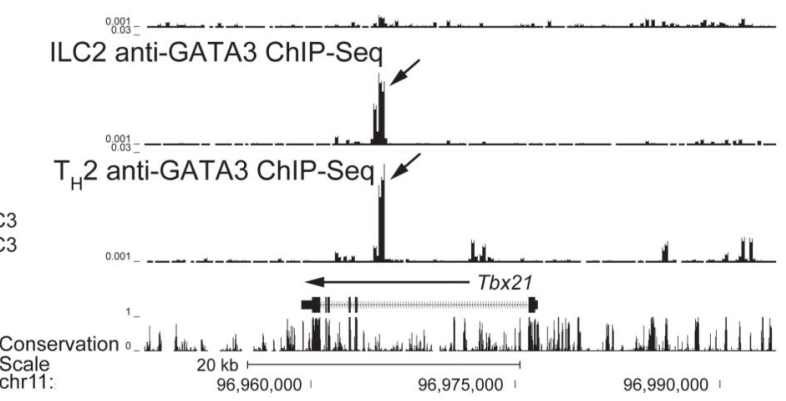

d

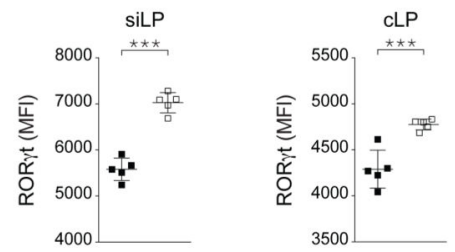

f

ILC3 anti-GATA3 ChIP-Seq

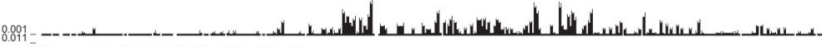

ILC2 anti-GATA3 ChIP-Seq

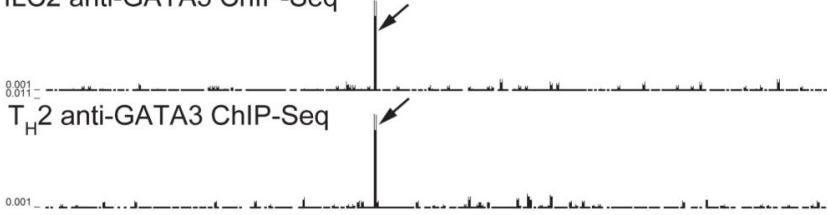

Rorc $\longrightarrow$

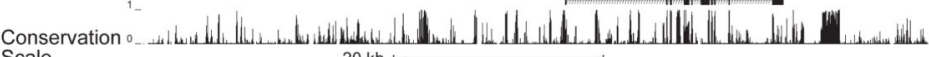
Scale

$94,160,000$

$94,200,000$

$\|$

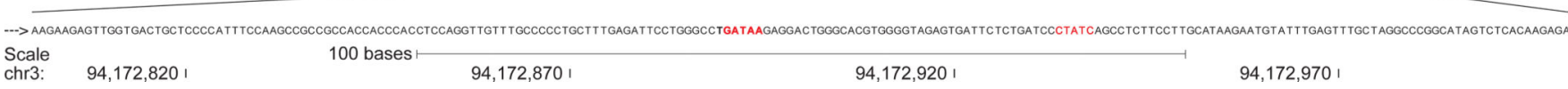

Figure 3.

GATA3 negatively regulates ROR $\gamma$ t without affecting T-bet expression. (a) Lymphocytes from the siLP of the $\operatorname{Gata}^{\mathrm{fl} / \mathrm{fl}}$ and $\operatorname{Gata}^{\mathrm{AILC} 3}$ mice $(\mathrm{n}=3)$ were prepared and stained as in Fig. 2a. Live lineage ${ }^{-}$cells were separated into different ILC3 populations by gating and Tbet expression of each subset is shown. T-bet staining in CCR6 ${ }^{+}$ILC3s was used as a negative control. (b) A UCSC genome browser screen shot showing the normalized antiGATA3 ChIP-Seq read density around $T b x 21$ from ILC3s, ILC2s and $\mathrm{T}_{\mathrm{H}} 2$ cells, with genomic region enriched with GATA3 binding highlighted by arrows. (c, d) Lymphocytes from the siLP and cLP of the Gata3 ${ }^{f 1 / f 1}$ and Gata $^{\Delta \mathrm{ILC} 3}$ mice $(\mathrm{n}=5)$ were prepared and stained as in a. ROR $\gamma \mathrm{t}$ level in siLP ILC3s of Gata $^{\mathrm{fl} / \mathrm{fl}}$ and Gata3 ${ }^{\Delta \mathrm{ILC} 3}$ mice was analyzed by flow cytometry (c). MFI of ROR $\gamma \mathrm{t}$ level in siLP and cLP ILC3s from Gata $^{\mathrm{fl} / \mathrm{fl}}$ and Gata3 $^{\Delta \mathrm{ILC} 3}$ mice was calculated $(\mathbf{d})$. (e) Gata $^{\mathrm{fl} / \mathrm{fl}} \mathrm{Cre}^{\mathrm{E}} \mathrm{ER}^{\mathrm{T} 2}$ mice (n=3 per group) were treated with tamoxifen every other day for three times. One week after the first injection, 
lymphocytes from siLP were harvested and stained as in Fig. 1c. ROR $\gamma t$ expression by siLP ILC3s from untreated and tamoxifen treated Gata $3^{\mathrm{fl} / \mathrm{fl}} \mathrm{Cre}-\mathrm{ER}^{\mathrm{T} 2}$ mice was analyzed. (f) A UCSC genome browser screen shot showing the normalized anti-GATA3 ChIP-Seq read density around Rorc from ILC3s, ILC2s and $\mathrm{T}_{\mathrm{H}} 2$ cells, with genomic region enriched with GATA3 binding highlighted by arrows. Two putative GATA3 binding motifs with consensus GATA3 binding sequence, as indicated by red color, were identified within the peak area. Each error bar represents s.d. of the mean. *** $P<0.001$ (two-tailed unpaired Student's $t$ test). Data are representative of at least three independent experiments $(\mathbf{a}, \mathbf{c}, \mathbf{d})$ and one experiment $(\mathbf{b}, \mathbf{e}, \mathbf{f})$. 
a

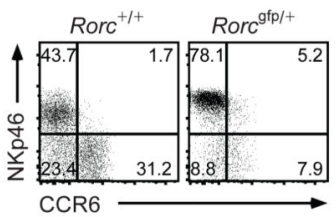

d

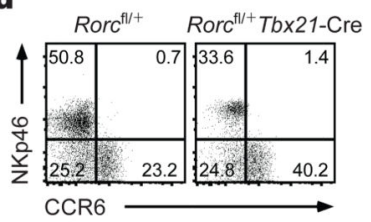

b

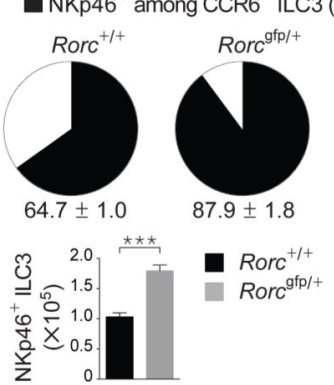

e

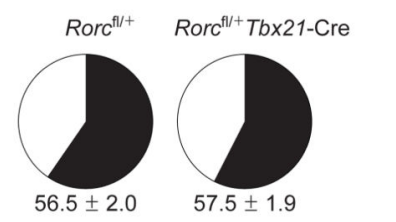

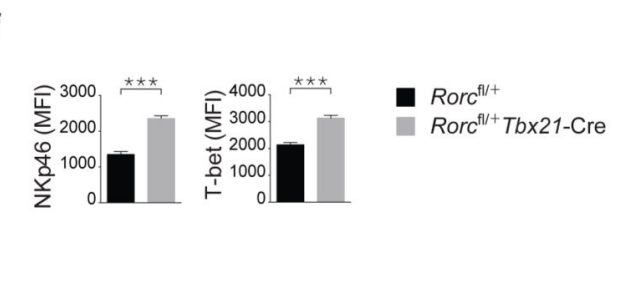

c

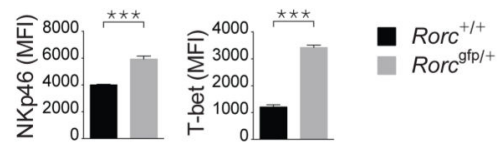

- $\mathrm{NKp} 6^{+}$among CCR6 ${ }^{-}$ILC3 (\%)

g

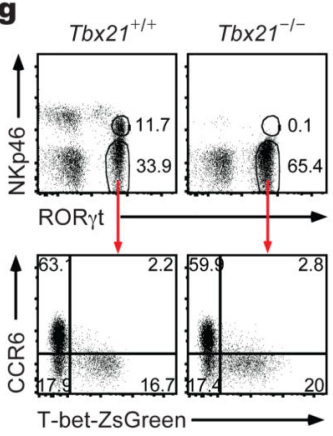

h

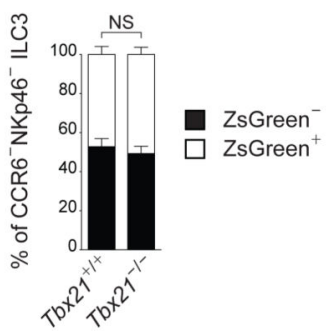

j

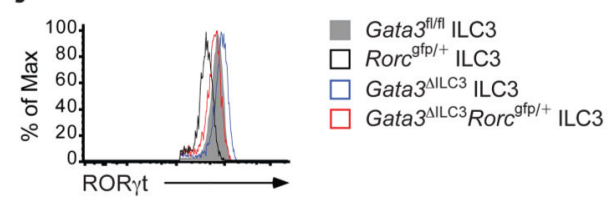

i

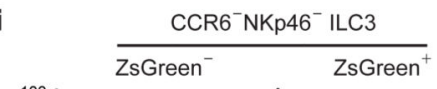

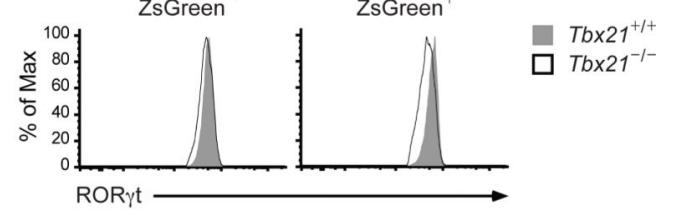

$\mathbf{k}$

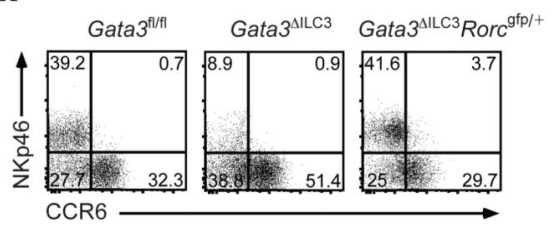

I
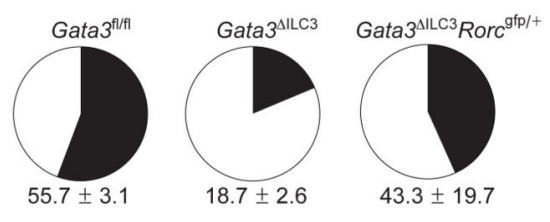

NKp $46^{+}$among CCR6 ${ }^{-}$ILC3 (\%)

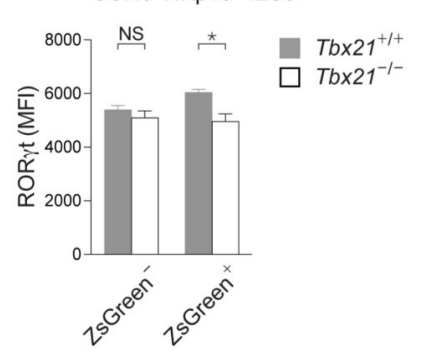

\section{Figure 4.}

The interplay among ROR $\gamma$ t, GATA3 and T-bet regulates the development of NKp $46^{+}$ILC3 at different stages. (a-c) Lymphocytes from the siLP of the Rorc $^{+/+}$and Rorc ${ }^{g f p /+}$ mice $(n=3)$ were stained as in Fig. 2a. Different subsets of ILC3s were analyzed by flow cytometry (a). The total NKp46 $6^{+}$ILC3 cell number and the frequency of NKp $46^{+}$ILC3s were calculated (b). The MFI of NKp46 expression within NKp46 ILC3s were compared (c). (d-f) Lymphocytes from the siLP of the $R o r c^{\mathrm{fl} /+}$ and $\operatorname{Rorc}^{\mathrm{fl} /+}$ Tbx21-Cre mice $(\mathrm{n}=2)$ were stained as in a. Different subsets of ILC3s were analyzed by flow cytometry (d). The 
frequency of NKp $46^{+}$ILC3 was calculated (e). The MFI of NKp46 expression within NKp $46^{+}$ILC3s were compared (f). (g, h, i) Lymphocytes from the siLP of the $T b \times 21^{+/+}$and $T b \times 21^{-1-}$ mice that also carrying T-bet-ZsGreen $(\mathrm{n}=3)$ were stained as in $\mathbf{a}$. Live lineage ${ }^{-}$ cells were separated into different ILC3 populations by gating and T-bet-ZsGreen reporter expression in NKp46- ILC3s is shown (g). Percentage of T-bet-ZsGreen ${ }^{+}$ILC3s within CCR6 $6^{-} \mathrm{NKp} 46^{-}$population was calculated (h). ROR $\gamma \mathrm{t}$ expression and MFI in ZsGreen ${ }^{-} \mathrm{CCR} 6^{-}{ }^{-N K p} 46^{-}$and $\mathrm{ZsGreen}{ }^{+} \mathrm{CCR} 6^{-} \mathrm{NKp} 46^{-}$ILC3s was analyzed (i). (j-l) Lymphocytes from the siLP of indicated mice ( $\mathrm{n}>=3$ per group) were stained as in $\mathbf{a}$. ROR $\gamma \mathrm{t}$ level was assessed by flow cytometry (j). Different subsets of ILC3s were analyzed by flow cytometry (k). The percentage of NKp46 ${ }^{+}$ILC3 within the CCR6 $6^{-}$ILC3 lineage was calculated (l). Each error bar represents s.d. of the mean. NS not significant, $* P<0.05$, $* * * P<0.001$ (two-tailed unpaired Student's $t$-test). Data are representative of at least three $(\mathbf{a}-\mathbf{c}, \mathbf{j}, \mathbf{k})$ and two $(\mathbf{d}-\mathbf{i})$ independent experiments or a combination of four experiments (l). 


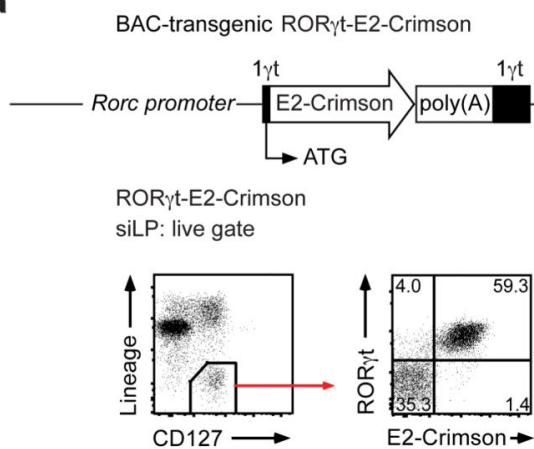

C

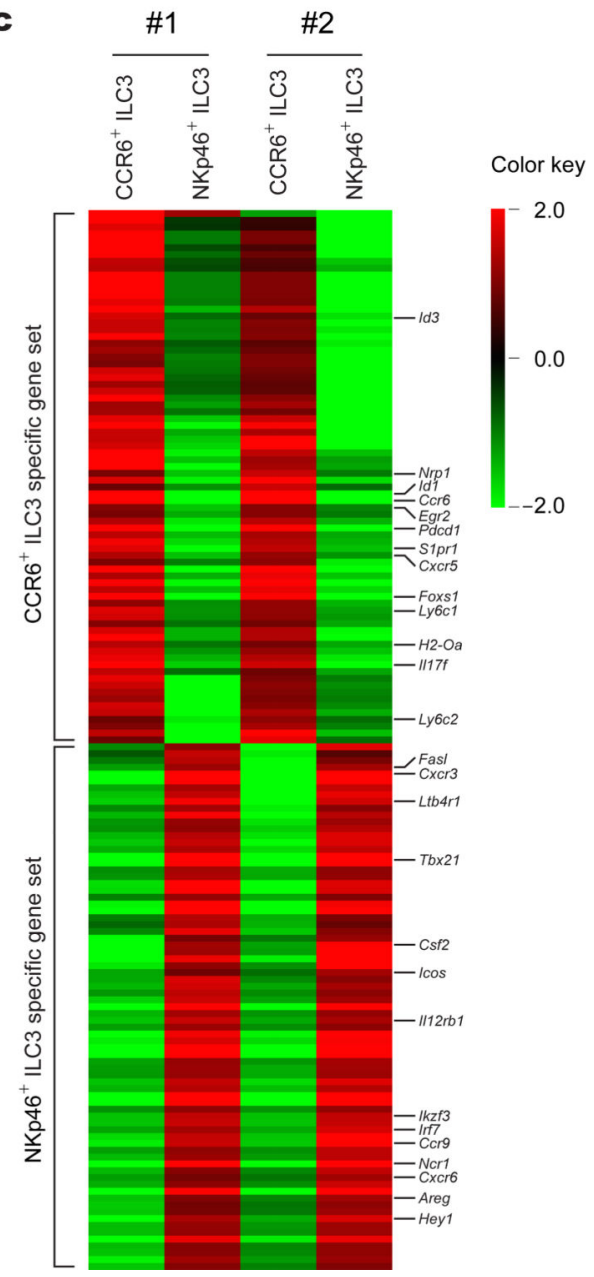

b
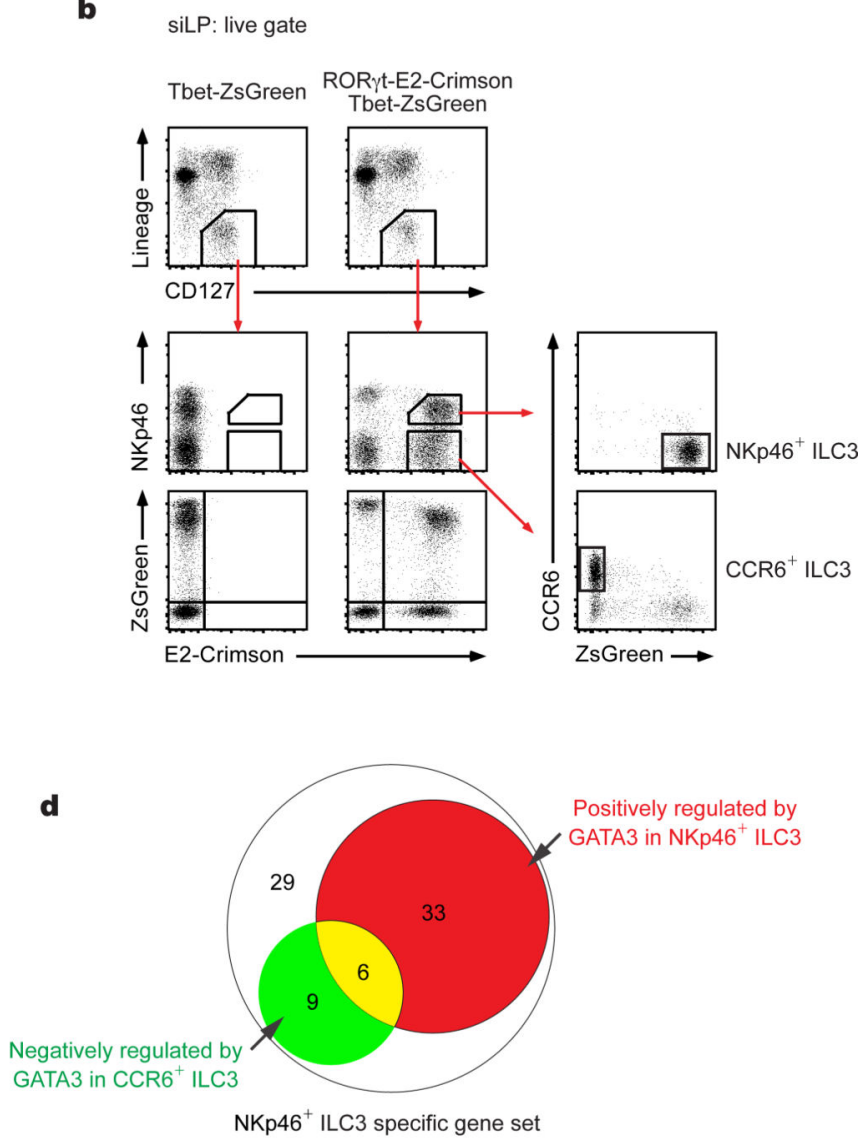

e

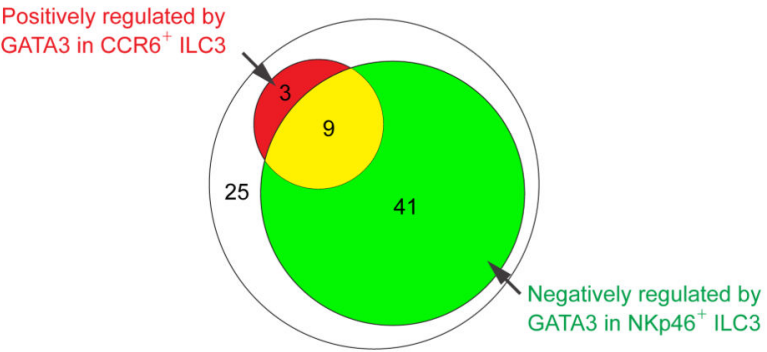

$\mathrm{CCR6}^{+}$ILC3 specific gene set

Figure 5.

GATA3 globally regulates lineage-preferred genes that are selectively expressed by CCR6 ${ }^{+}$ or NKp46 ${ }^{+}$ILC3 subsets. (a) Map of the ROR $\gamma$ t-E2-Crimson BAC construct used for making transgenic mice. Lymphocytes from the siLP of the ROR $\gamma \mathrm{t}-\mathrm{E} 2-\mathrm{Crimson} \mathrm{BAC}$ transgenic reporter were stained as in Fig. 1a. Co-expression of ROR $\gamma \mathrm{t}$ and E2-Crimson in ILCs (live lineage ${ }^{-}$CD $127^{+}$) were analyzed by flow cytometry. (b) Lymphocytes from the siLP of the T-bet-ZsGreen and ROR $\gamma$ t-E2-Crimson dual-reporter mice were stained as in Fig. 2a. T-bet-ZsGreen single reporter mice were taken as negative control for ROR $\gamma \mathrm{t}-\mathrm{E} 2-$ 
Crimson. Lineage ${ }^{-} \mathrm{CD} 127^{+} \mathrm{E} 2-\mathrm{Crimson}^{+} \mathrm{NKp} 46^{+} \mathrm{ZsGreen}^{+} \mathrm{CCR} 6^{-}$and lineage ${ }^{-} \mathrm{CD} 127^{+} \mathrm{E} 2-$ Crimson ${ }^{+} \mathrm{NKp} 46^{-} \mathrm{ZsGreen}^{-} \mathrm{CCR} 6^{+}$populations were gated to guide cell sorting for $\mathrm{NKp} 46^{+}$ and $\mathrm{CCR} 6^{+}$ILC3s, respectively. (c, d, e) RNA-Seq analyses were carried out with sorted NKp $46^{+}$and CCR6 ${ }^{+}$ILC3s as illustrated in $\mathbf{b}$ from siLP of the Gata $3^{\mathrm{fl} / \mathrm{fl}}$ and Gata3 $3^{\mathrm{\Delta ILC} 3}$ that also carried T-bet-ZsGreen and ROR $\gamma$ t-E2-Crimson dual-reporter. Heatmap showing the 77 and 78 genes that were preferentially expressed by NKp46 ${ }^{+}$and CCR6 $6^{+}$ILC3s, respectively (fold change $>4$, RPKM value $>10$ in either sample). Several representative genes were highlighted (c) Venn diagram showing the $77 \mathrm{NKp} 46^{+}$ILC3 specific genes that were positively regulated ( $>2$ fold) by GATA3 in NKp46 $6^{+}$ILC3s and/or negatively regulated (> 2 fold) by GATA3 in CCR6 ${ }^{+}$ILC3s (d). Venn diagram showing the 78 CCR6 $6^{+}$ILC3 specific genes that were positively regulated (> 2 fold) by GATA3 in CCR6 ${ }^{+}$ILC3s and/or negatively regulated (> 2 fold) by GATA3 in NKp46 ${ }^{+}$ILC3s (e). Data are representative of one experiment with duplicates. 
a

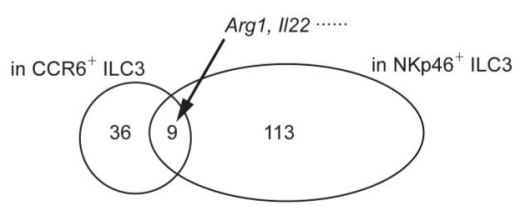

GATA3 positively regulated genes

b

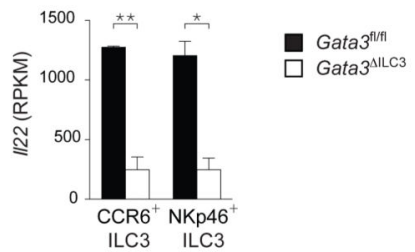

C

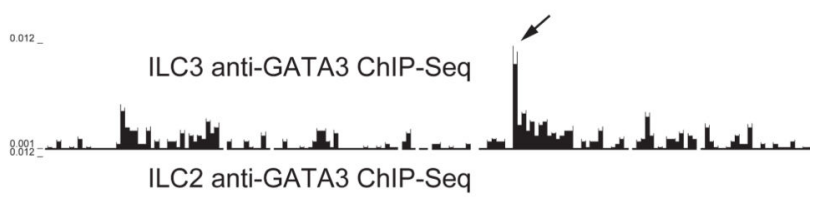

0.091

$\mathrm{T}_{\mathrm{H}} 2$ anti-GATA3 ChIP-Seq

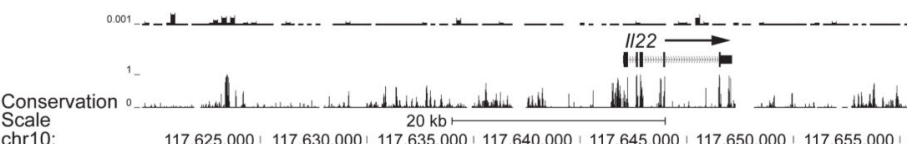

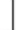

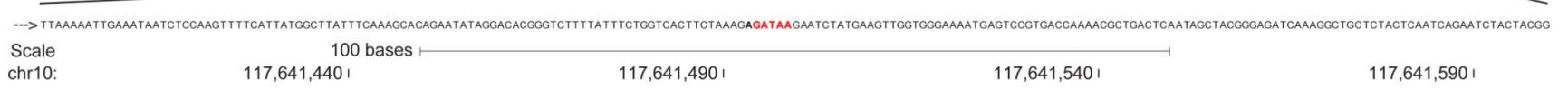

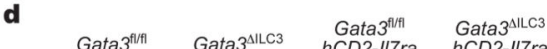
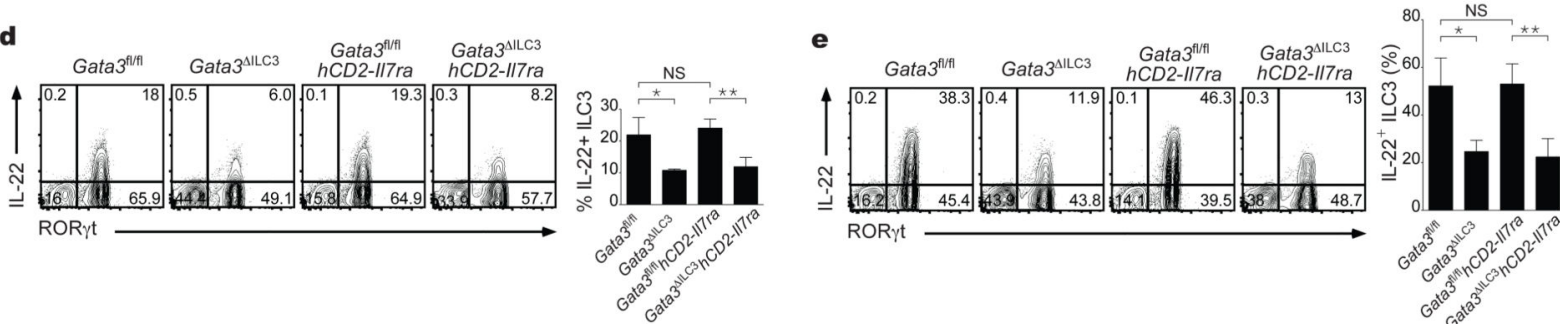

f

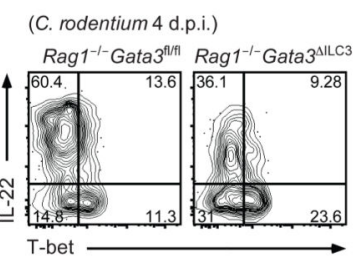

$\mathbf{g}$
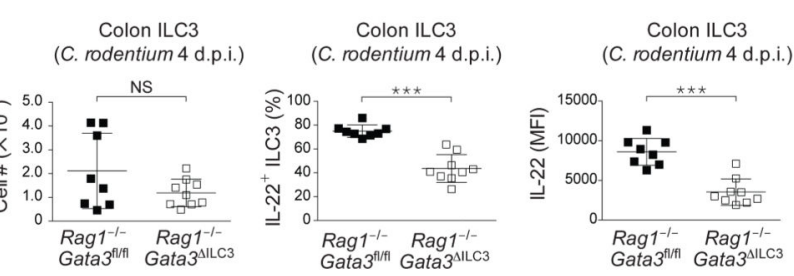

i

h
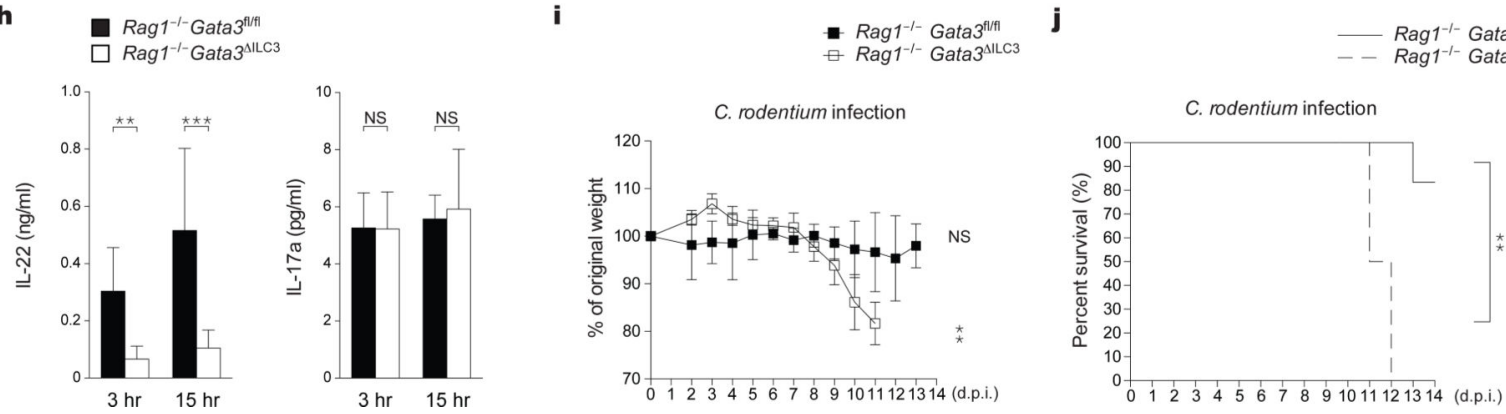

Figure 6.

GATA3 regulates IL-22 production by ILC3s and thus susceptibility of mice to $C$. rodentium infection. (a) Venn diagram showing genes that were positively regulated by GATA3 in ILC3 subsets. (b) Bar graph showing alteration of II22 mRNA (based on RPKM values from RNA-Seq) in ILC3s upon Gata3 removal. c, A UCSC genome browser screen shot showing the normalized anti-GATA3 ChIP-Seq read density around $I I 22$ from indicated cells. (d) Lymphocytes from siLP of the indicated mice ( $\mathrm{n}=3$ per group) were cultured in vitro for 3 hours. Production of IL-22 by ILCs was analyzed by flow cytometry and the percentage of 
IL-22 ${ }^{+}$ILC3s among ROR $\gamma \mathrm{t}^{+}$cells was plotted. (e) Similar analyses were carried out as in d, except for the cells were treated with IL-23 for 3 hours. (f-h) Gata $3^{\mathrm{Il} / \mathrm{fl}}(\mathrm{n}=4)$ and Gata $^{\mathrm{\Delta ILC} 3}$ $(\mathrm{n}=5)$ mice on the $R a g 1^{-1-}$ background were infected with $C$. rodentium. IL-22 production and T-bet expression by cLP ILC3s were analyzed ex vivo 4 days after infection (f). ILC3 total cell number, percentage of IL-22-producing cells within ILC3s, and IL-22 MFI within IL-22+ ILC3s were calculated (g). The amount of IL-22 and IL-17a in the supernatant of cultured cLP cells was analyzed with multiplex cytokine assay (h). (i, j) $\operatorname{Rag}^{-{ }^{-}-}$Gata3 $^{\mathrm{AILC} 3}$ $(\mathrm{n}=4)$ and $\operatorname{Rag}^{-/-}{ }^{-}$Gata $^{\mathrm{fl} / \mathrm{fl}}(\mathrm{n}=6)$ mice were infected with $C$. rodentium. Weight loss (i) and survival rate (j) of $\operatorname{Rag}^{1^{-/}}$Gata3 $^{\text {fl/fl }}$ and $\operatorname{Rag}^{-{ }^{-}-} \mathrm{Gata}^{\mathrm{AILC} 3}$ was monitored. Each error bar represents s.d. of the mean. NS not significant, $* P<0.05$, ** $P<0.01$, *** $P<0.001$ (two-tailed unpaired Student's $t$-test, except for $\mathbf{i}$ with one way ANOVA and $\mathbf{j}$ with two-way ANOVA). Data are representative of at least three (d, e), two (f) independent experiments and one experiment $(\mathbf{a}-\mathbf{c}, \mathbf{i}, \mathbf{j})$ or a combination of two experiments $(\mathbf{g}, \mathbf{h})$. 